Paper published in Nat. Mater. 16, 987 (2017); doi:10.1038/nmat4963.

\title{
Bronze-mean hexagonal quasicrystal
}

Tomonari Dotera, ${ }^{1}$ Shinichi Bekku, ${ }^{1}$ and Primož Ziherl ${ }^{2,3}$

${ }^{1}$ Department of Physics, Kindai University,

3-4-1 Kowakae Higashi-Osaka 577-8502, Japan

${ }^{2}$ Faculty of Mathematics and Physics, University of Ljubljana,

Jadranska 19, SI-1000 Ljubljana, Slovenia

${ }^{3}$ Jožef Stefan Institute, Jamova 39, SI-1000 Ljubljana, Slovenia

(Dated: July 4 2017) 
The most striking feature of conventional quasicrystals is their nontraditional symmetry characterised by icosahedral, dodecagonal, decagonal, or octagonal $\operatorname{axes}^{1-6}$. The symmetry and the aperiodicity of these materials stem from an irrational ratio of two or more length scales controlling their structure, the best-known examples being the Penrose ${ }^{7,8}$ and the Ammann-Beenker ${ }^{9,10}$ tiling as two-dimensional models related to the golden and the silver mean, respectively. Surprisingly, no other metallic-mean tilings have been discovered so far. Here we propose a self-similar bronze-mean hexagonal pattern, which may be viewed as a projection of a higher-dimensional periodic lattice with a Koch-like snowflake projection window. We use numerical simulations to demonstrate that a disordered variant ${ }^{11}$ of this quasicrystal can be materialised in soft polymeric colloidal particles with a core-shell architecture ${ }^{12-17}$. Moreover, by varying the geometry of the pattern we generate a continuous sequence of structures, which provide an alternative interpretation of quasicrystalline approximants observed in several metal-silicon alloys ${ }^{18}$.

The golden ratio has been pondered since ancient times in arts, architecture, mathematics, and science. Its key modern manifestation through Penrose tiles (Fig. 1a) and the discovery of quasicrystals (QCs) led to a paradigm shift, initiating a new field of research in both solidstate and soft-matter physics as well as in materials science. The golden ratio (golden mean hereafter) is hidden in the geometry of the regular pentagon and in the ratio of consecutive Fibonacci numbers characteristic of sequences generated by simple substitution rules. In their generalised form ${ }^{19,20}$, these rules read $\left\{\mathrm{A} \rightarrow \mathrm{A}^{k} \mathrm{~B}, \mathrm{~B} \rightarrow \mathrm{A}\right\}$ so that the number of $\mathrm{As}$ in the $n$-th generation denoted by $A_{n}$ is determined by the recurrence relation

$$
\left(\begin{array}{c}
A_{n+1} \\
A_{n}
\end{array}\right)=\left(\begin{array}{ll}
k & 1 \\
1 & 0
\end{array}\right)\left(\begin{array}{c}
A_{n} \\
A_{n-1}
\end{array}\right) .
$$

The inflation factors defined by $A_{n+1} / A_{n}$ at large $n$ are given by $\left(k+\sqrt{k^{2}+4}\right) / 2$ and referred to as the metallic means (Fig. 1b).

The Fibonacci $k=1$ sequence related to the Penrose tiling and icosahedral QCs is the most famous pattern generated by these rules, its inflation factor being the golden mean (Fig. 1b). Also well-studied is the silver-mean $k=2$ sequence underlying the AmmannBeenker octagonal tiling (Fig. 1a and Supplementary Fig. S1). But the $k=3$ sequence 
based on the bronze mean

$$
\beta=\frac{3+\sqrt{13}}{2}=3+\frac{1}{3+\frac{1}{3+\frac{1}{\cdots}}} \approx 3.303
$$

remains elusive. No associated tiling is known nor it can be constructed in the same way as Penrose and Ammann-Beenker tilings because there exist no regular polygon with a diagonal-to-edge ratio equal to $\beta$ [ref. $\left.{ }^{21}\right]$.

Here we present a self-similar bronze-mean tiling composed of three types of tiles based on two lengths: Small (ST) and large (LT) equilateral triangles of edge length $s$ and $\ell$, respectively, and $\ell \times s$ rectangles $(\mathrm{R})$ (Fig. 1a). The fundamental motif of the tiling is a 6-fold-symmetric irregular dodecagon consisting of 6 copies of each tile (Fig. 1c). In each inflation step, the tiles are subdivided according to rules in Fig. 1a. Equivalently, the nextgeneration tiling can be obtained by magnifying the previous-generation tiling by the bronze mean and then placing the fundamental dodecagons at its vertices as visualised by Fig. 1d (Supplementary Movie 1).

A patch of the dodecagon containing $1 \mathrm{ST}, 1 \mathrm{LT}$, and $1 \mathrm{R}$ tile in Fig. $1 \mathrm{~d}$ is subdivided twice, showing a part of the third-generation tiling. By inspecting it, we observe that the long lengths of three consecutive generations $\ell_{n+1}, \ell_{n}$ and $\ell_{n-1}$ satisfy Eq. (1) with $k=3$; this also holds for the short lengths $s_{n}$ (Fig. 1f). The inflation factor is equal to the bronze mean $\beta$ and the corresponding edges of the three outlined rectangles in Fig. 1d are magnified by $\beta$ in each generation.

All generations of the bronze tiling are self-similar in the sense that they contain the original pattern in the centre (Fig. 1d \& Supplementary Fig. S2). Vertices of a given generation lie on sets of parallel lines (Fig. 1d) such that the spacings between them constitute two variants of the bronze-mean sequence generated by rules $\{\mathrm{A} \rightarrow \mathrm{AABA}, \mathrm{B} \rightarrow \mathrm{A}\}$ and $\{\mathrm{C} \rightarrow \mathrm{CDC}, \mathrm{D} \rightarrow \mathrm{CDCC}\}$. In these substitutions, the lengths $\ell_{n}$ and $s_{n}$ transform as

$$
\left(\begin{array}{l}
\ell_{n+1} \\
s_{n+1}
\end{array}\right)=\left(\begin{array}{cc}
2 & \sqrt{3} \\
\sqrt{3} & 1
\end{array}\right)\left(\begin{array}{l}
\ell_{n} \\
s_{n}
\end{array}\right) .
$$

The larger eigenvalue of this matrix is again $\beta$, the corresponding eigenvector $((\sqrt{3}+\sqrt{39}) / 6,1) \approx$ $(1.330,1)$ giving the exact self-similar length ratio $\ell / s$. The numbers of small triangles, large triangles, and rectangles of $(n+1)$-th generation denoted by $S T_{n+1}, L T_{n+1}$, and $R_{n+1}$, re- 
spectively, are given by

$$
\left(\begin{array}{c}
S T_{n+1} \\
L T_{n+1} \\
R_{n+1}
\end{array}\right)=\left(\begin{array}{ccc}
1 & 3 & 4 \\
3 & 4 & 8 \\
\frac{3}{2} & 3 & 5
\end{array}\right)\left(\begin{array}{c}
S T_{n} \\
L T_{n} \\
R_{n}
\end{array}\right)
$$

The components of the normalised principal eigenvector of the transition matrix give the ideal frequencies of ST, LT, and R tiles which $\mathrm{read}(29-5 \sqrt{13}) / 43 \approx 0.255,(23-\sqrt{13}) / 43 \approx$ 0.451 , and $(-9+6 \sqrt{13}) / 43 \approx 0.294$, respectively. These irrational numbers mean that the bronze tiling cannot possibly be periodic.

The bronze tiling bears a superficial similarity to the Stampfli square-triangle dodecagonal tiling $^{22}$ (Supplementary Discussion II) but there are two fundamental distinctions between them. First, even if we start with a length ratio $\ell_{1} / s_{1}=1$ so that the first-generation ST and LT tiles are identical and the R tile is a square, Eq. (3) ensures that in the late generations $\ell_{n} / s_{n}$ tends to 1.330 and that these tilings approach the self-similar bronze tiling. Indeed, Fig. 1e shows that after a single inflation where we assemble squares and identical triangles into second-generation ST, LT, and R tiles according to bronze subdivision rules in Fig. 1a, the length ratio of these tiles is $\ell_{2} / s_{2} \approx 1.366$. Using a straightedge, one can easily see that these second-generation tiles are almost indistinguishable from their ideal counterparts immediately above in Fig. 1a. Second, in the Stampfli tiling every dodecagon can be independently rotated by $30^{\circ}$ but in the bronze tiling only $60^{\circ}$ rotations are possible (Figs. 1g and h). Here the edges lie along 12 in-plane directions divided into alternating sets of 6 , one for the short and the other for the long edges; hence the 6-fold symmetry.

Equally interesting as the ideal bronze tiling are its non-self-similar modifications with $\ell / s$ different from 1.330 (Fig. 1i). If the ratio is either zero or infinite, this pattern reduces to a hexagonal tiling consisting solely of ST and LT tiles, respectively, and for $\ell / s=1$ it is the 6-fold square-triangle tiling. Here the two types of edges can be distinguished by atomic decorations on midpoints (open and filled circles in Fig. 1f), typically realised in the Frank-Kasper phases $^{18}$. Also possible are three related patterns based on ST, LT, and R tiles. In the non-self-similar pattern in Fig. 1j the first-generation ST tiles form the central hexagonal rosette, the $\mathrm{R}$ tiles are radially arranged around it, and the LT tiles fill the gaps between the $\mathrm{R}$ tiles. Patterns in Figs. $1 \mathrm{k}$ and $\mathrm{l}$ are the high density version of those in Figs. $1 \mathrm{j}$ and $\mathrm{d}$, respectively, both obtained by swapping ST and LT tiles and rotating R tiles by $90^{\circ}$. 
In practice, the generalised bronze patterns may be more relevant than the self-similar tiling itself because together with their disordered versions, they constitute a broad class of structures which may be easier to fabricate or identify. To demonstrate that a simple pair interaction suffices for the formation of an almost ideal random bronze tiling, we performed Monte-Carlo simulations of a 2D ensemble of hard-core/square-shoulder (HCSS) disks characterised by core and shoulder diameter $\sigma$ and $\lambda \sigma$, respectively, and by shoulder height $\varepsilon$ (Fig. 2a). The step-like HCSS interaction is particularly suitable because in the ideal bronze tiling, the two next-nearest-neighbour distances $\sqrt{s^{2}+\ell^{2}+\sqrt{3} s \ell}$ and $\sqrt{3} \ell$ (green and violet edge in Fig. 2b, respectively) differ by just $2.2 \%$, which is unlikely to show in simulations. Thus we slightly adjust the $\ell / s$ ratio such that the two distances are identical, and we tune the shoulder width so that it reaches exactly to next-nearest neighbours. Given the natural choice $s=\sigma$, the shoulder-to-core ratio satisfying this condition is $\lambda=(3+\sqrt{33}) / 4 \approx 2.186$. This value of $\lambda$ simultaneously stabilises the ST-R and LT-R pairs and the LT-LT motif in the dodecagonal and the rugby-ball patterns (Fig. 2b).

A snapshot of a bronze-mean quasicrystal (BMQC) obtained by annealing a quenched ensemble of $\lambda=2.18$ HCSS particles of packing fraction $\eta=0.595$ (Methods, Supplementary Discussion III and Supplementary Movie 2) is shown in Fig. 2c. A mosaic of ST, LT, and R tiles forms many aligned bronze-tiling dodecagonal patterns whose centres are indicated by blue circles. The bond-orientational order parameters indicate 6-fold symmetry (Supplementary Fig. S5) and the tile frequencies all approach the ideal BMQC values (Fig. 3).

The Fourier transform of particles' centres of mass in Fig. 2e is marked by 6-fold discrete peaks as well as by self-similar patterns of hexagons and rectangles. Evidently, these peaks cannot be indexed by two integers, which is attributed to aperiodicity. The real-space snapshot contains several patches which can be viewed as basic approximants of the bronze tiling (Fig. 2d). By examining the approximants, we see that their transforms do capture certain features of the BMQC transform in Fig. 2e but none of them completely agrees with it (Supplementary Discussion IV).

To better understand the BMQC, we employ the higher-dimensional analysis. Here a quasicrystalline structure such as the Penrose tiling is represented by a crystal in higherdimensional superspace and its actual form is viewed as a projection of this crystal onto the $2 \mathrm{D}$ physical space ${ }^{5,6}$. In this powerful method, the superspace is divided into the physical 
space and its complement known as the perpendicular space, and the projection of lattice points on the latter is referred to as the window.

We construct the window corresponding to the self-similar bronze tiling by mapping the vertices of the first- to fifth-generation tilings to the perpendicular space. The sequence of the obtained windows in Fig. 4a-e and especially its elaborate fifth-generation member generated from 324391 points show that the bronze-tiling projection window is fractal, its shape being the Koch-like snowflake ${ }^{24}$. This fractal window allows us to exactly evaluate the Fourier transform of the self-similar bronze tiling, which coincides rather well with the transform of the random BMQC obtained in simulations (Fig. 2e); the details of the higherdimensional analysis including a comparison of peak positions and intensities can be found in Supplementary Discussion V.

The packing fraction in Fig. 2c is somewhat smaller than the ideal value of $\eta_{\mathrm{BMQC}}=0.641$ (Supplementary Table SIII) which allows the system to equilibrate by particle rearrangements. Rearrangements are mediated by shield-shaped holes as sketched in Fig. 5. Consider a part of the ideal bronze tiling with two 2-tile wide regions coloured grey, their highlighted backbones running off-centre. At a lower-than-ideal packing fraction, not all vertices are occupied. For example, in the larger grey region in Fig. 5a packing fraction can be decreased by removing a tip particle as shown in Fig. 5b. This creates a hole in the tiling and allows the neighbouring particle along the backbone to move to the nearby energetically equivalent position within the hole. The hole is thus divided into two and one of them propagates towards the other tip of the region in a sequence of a zipper moves ${ }^{11}$, which can also be viewed as local flips of lattice planes ${ }^{23}$ (Fig. 5c). As a result, the initially self-similar pattern is disordered and a shield-shaped hole appears at either tip of the backbone. These holes cannot be filled by the ST, LT, and R tiles, indicating that adding particles in the holes is energetically unfavorable.

To check the stability of the BMQC formed by HCSS particles with $\lambda=2.18$, we carried out numerical thermalisation experiments in a system of 14389 particles forming a rectangle approximant with $\eta=0.600$ (Supplementary Information VI). Particle positions in the perpendicular space after thermalisation at $k_{\mathrm{B}} T / \varepsilon=0.20$ and $k_{\mathrm{B}} T / \varepsilon=0.25$ are shown in Figs. $4 \mathrm{~b} \& \mathrm{c}$, respectively. At the lower temperature, the positions almost perfectly agree with the snowflake fractal window which shows that the system is stabilised energetically are locked in an almost perfect-tiling state ${ }^{25}$, whereas at the higher temperature it is entropically 
stabilised in a random-tiling state. Compared to the one-generation-smaller approximant with 1319 particles, the phason fluctuations increase with system size at $k_{\mathrm{B}} T / \varepsilon>0.22$ consistent with the entropic ordering mechanism (Supplementary Fig. S16) ${ }^{11}$.

The generalised bronze tiling may be viewed as a model for QC approximants seen in metal-silicon alloys based on $\mathrm{Mn}$ and $\mathrm{Cr}$, Ni, or $\mathrm{V}^{18,26,27}$, which were so far associated with the square-triangle dodecagonal tiling. Yet the structural models of the Frank-Kasper phases show that in terms of chemical composition of each layer, the square tiles in the hexagonal approximant (or A15 structure) do not have the 4-fold symmetry and the two types of triangular tiles are inequivalent like in Fig. 1e. In these alloys, the non-self-similar ST, LT, and R tiles have been identified in real-space electron micrographs [Fig. 10 of ref. ${ }^{18}$ ]; also consistent with the generalised bronze tiling is the virtually uniform orientation of dodecagons. In addition, the non-self-similar tiles were seen in a QC approximant reported in ultrathin $\mathrm{BaTiO}_{3}$ films ${ }^{28}$ as well as in quasiperiodic and periodic superlattices formed in a dried binary nanocrystal solution ${ }^{29}$. These reports suggests that the bronze tiling itself may well be expected in suitably prepared samples based on alloys, oxides, or nanoparticles.

Our BMQC re-establishes that QCs can have one of the common rotational symmetries ${ }^{30}$. For soft-matter systems which usually produce only a few diffraction peaks, this is an important message because a 6-fold symmetric diffraction pattern is routinely regarded as a strong indication of periodic hexagonal order, and we show that this is not necessarily justified. Also relevant for materials science is the proposed physical model of BMQC which provides clues to the rational design of the novel quasicrystal in polymeric nanocolloidal suspensions ${ }^{12-14}$ and extends the rich spectrum of ordered structures promoted by the underlying two-lengthscale force ${ }^{17}$.

1 Levine, D. \& Steinhardt, P. J. Quasicrystals: A new class of ordered structures. Phys. Rev. Lett. 53, 2477-2480 (1984).

2 Shechtman, D., Blech, I., Gratias, D. \& Cahn, J. W. Metallic phase with long-range orientational order and no translational symmetry. Phys. Rev. Lett. 53, 1951-1953 (1984).

3 Ishimasa, T., Nissen, H.-U. \& Fukao, Y. New ordered state between crystalline and amorphous in Ni-Cr particles. Phys. Rev. Lett. 55, 511-513 (1985). 
4 Tsai, A. P., Inoue, A. \& Masumoto, T. A stable quasicrystal in Al-Cu-Fe system. Jpn. J. Appl. Phys. 26, L1505-L1507 (1987).

5 Janssen, T., Chapuis, G. \& de Boissieu, M. Aperiodic Crystals: From Modulated Phases to Quasicrystals (Oxford University Press, 2007).

6 Steurer, W. \& Deloudi, S. Crystallography of Quasicrystals: Concepts, Methods and Structures (Springer, 2009).

7 Penrose, R. The role of aesthetics in pure and applied mathematical research. Bull. Inst. Math. Appl. 10, 266-271 (1974).

8 Mackay, A. Crystallography and the Penrose pattern. Physica A 114, 609-613 (1982).

9 Grünbaum, B. \& Shephard, G. C. Tilings and Patterns (Freeman, 1987).

10 Beenker, F. P. M. Algebraic theory of non-periodic tilings of the plane by two simple building blocks: a square and a rhombus. TH-Report 82-WSK04, 1-64 (Eindhoven University of Technology, 1982).

11 Oxborrow, M. \& Henley, C. L. Random square-triangle tilings: a model for twelvefold-symmetric quasicrystals. Phys. Rev. B 48, 6966-6998 (1993).

12 Zeng, X. et al. Supramolecular dendritic liquid quasicrystals. Nature 428, 157-160 (2004).

13 Chanpuriya, S. et al. Cornucopia of nanoscale ordered phases in sphere-forming tetrablock terpolymers. ACS Nano 10, 4961-4972 (2016).

14 Fischer, S. et al. Colloidal quasicrystals with 12-fold and 18-fold diffraction symmetry. Proc. Natl. Acad. Sci. USA 108, 1810-1814 (2011).

15 Iacovella, C. R., Keys, A. S. \& Glotzer, S. C. Self-assembly of soft-matter quasicrystals and their approximants. Proc. Natl. Acad. Sci. USA 108, 20935-20940 (2011).

16 Engel, M. \& Trebin, H.-R. Self-assembly of monatomic complex crystals and quasicrystals with a double-well interaction potential. Phys. Rev. Lett. 98, 225505 (2007).

17 Dotera, T., Oshiro, T. \& Ziherl, P. Mosaic two-lengthscale quasicrystals. Nature 506, 208-211 (2014).

18 Ishimasa, T. Dodecagonal quasicrystals still in progress. Isr. J. Chem. 51, 1216-1225 (2011).

19 Gumbs, G. \& Ali, M. K. Dynamical maps, Cantor spectra, and localization for Fibonacci and related quasiperiodic lattices. Phys. Rev. Lett. 60, 1081-1084 (1988).

20 Suzuki, T.-K. \& Dotera, T. Dynamical systems for quasiperiodic chains and new self-similar polynomials. J. Phys. A: Math. Gen. 26, 6101-6113 (1993). 
21 Buitrago, A. R. Polygons, diagonals, and the bronze mean. Nexus Netw. J. 9, 321-326 (2008).

22 Stampfli, P. Dodecagonal quasiperiodic lattice in two dimensions. Helv. Phys. Acta 59, 1260$1263(1986)$.

23 Socolar, J. E. S., Lubensky, T. C. \& Steinhardt, P. J. Phonons, phasons, and dislocations in quasicrystals. Phys. Rev. B 34, 3345-3360 (1986).

24 Niizeki, K. A step toward an MLD classification of selfsimilar quasilattices. Prog. Theor. Phys. 128, 629-691 (2012).

25 Dotera, T. \& Steinhardt, P. J. Ising-like transition and phason unlocking in icosahedral quasicrystals. Phys. Rev. Lett. 72 1670-1673 (1994).

26 Iga, H., Mihalkovič, M. \& Ishimasa, T. Approximant of dodecagonal quasicrystal formed in MnSiV alloy. Philos. Mag. 91, 2624-2633 (2011).

27 Ishimasa, T., Iwami, S., Sakaguchi, N., Oota, R. \& Mihalkovič, M. Phason space analysis and structure modelling of $100 \AA$ A-scale dodecagonal quasicrystal in Mn-based alloy. Philos. Mag. 95, 3745-3767 (2015).

28 Förster, S. et al. Observation and structure determination of an oxide quasicrystal approximant. Phys. Rev. Lett. 117, 095501 (2016).

29 Ye, X. et al. Quasicrystalline nanocrystal superlattice with partial matching rules. Nat. Mater. 16, 214-219 (2017).

30 Sasisekharan, V., Baranidharan, S., Balagurusamy, V. S. K., Srinivasan, A., \& Gopal, E. S. R. Non-periodic tilings in 2-dimensions with 4, 6, 8, 10 and 12-fold symmetries. Pramana - J. Phys. 33, 405-420 (1989).

Acknowledgements This project has been supported by Japan Society for the Promotion of Science through Grant-in-Aid for Scientific (B) (No. 16H04037), and has received funding from the European Union's Horizon 2020 research and innovation programme under the Marie Skłodowska-Curie grant agreement No. 642774. The authors acknowledge the financial support from the Slovenian Research Agency (research core funding No. P1-0055).

Author contributions T.D. and P.Z. conceived the project and proposed the tiling theory. S.B and T.D. performed simulations and higher-dimensional analysis, and T.D. and P.Z. wrote the manuscript. 
Additional information Supplementary information is available in the online version of the paper. Reprints and permissions information is available online at www . nature . com/reprints. Correspondence and requests for materials should be addressed to T.D. (email: dotera@phys.kindai.ac.jp)

Competing financial interests The authors declare no competing financial interests.

Caption to Supplementary Movie 1 Animation showing two subdivisions of the bronze dodecagonal pattern, leading to the third-generation tiling.

Caption to Supplementary Movie 2 Sequence of snapshots of a quarter of the $N=4000$ hard-core/square-shoulder ensemble at $\lambda=2.18$ initially quenched at $k_{B} T / \epsilon=0.20$ and then undergoing annealing at $k_{B} T / \epsilon=0.25$. Left panel shows raw snapshots with the cores of particles at the $3^{6}$ and $3^{2} .4 .3 .4$ vertices coloured blue and yellow whereas the cores of particles around shield-shaped defects are coloured red. In the right panel, second-generation tiles are highlighted.

\section{Methods}

Simulations. We used NVT Monte Carlo simulations of $N=4000$ particles confined to a square box of area $\mathcal{A}$ with periodic boundary conditions (more details in Supplementary Discussion III); the packing fraction of particles is defined by $\eta=\pi N \sigma^{2} / 4 \mathcal{A}$. Starting from a random state, all runs consisted of a quenching stage at a reduced temperature $k_{\mathrm{B}} T / \varepsilon=0.20$ needed so as to avoid metastable states and a slow annealing stage at a somewhat higher reduced temperature $k_{\mathrm{B}} T / \varepsilon=0.25$. The typical number of steps was between 1 and $3 \times 10^{9}$ as appropriate, with similar durations of the two stages. During simulation, we sampled energy and various structural quantities including bond-orientational order parameters and frequencies of ST, LT, and R tiles. The simulated diffraction patterns in Fig. $2 \mathrm{e}$ and in Fig. S13 are averaged Fourier intensities of positions of the particles. 


\section{Data and code availability}

The data that support the findings of this paper and the code used to generate it are available from the corresponding author on reasonable request. 
a

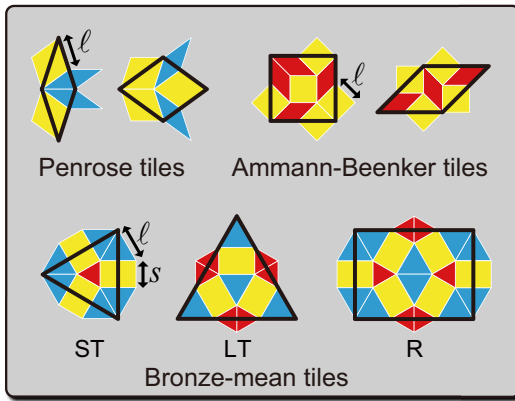

e

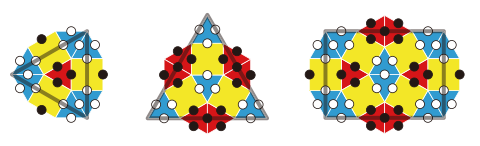

f

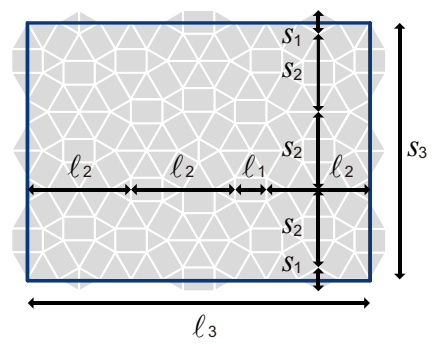

g

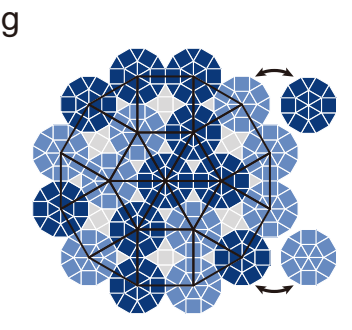

h

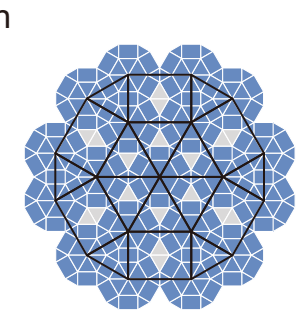

j

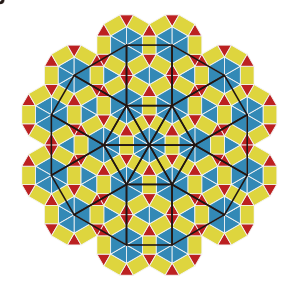

d b

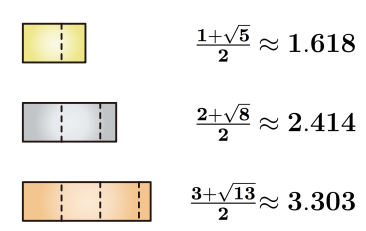

C
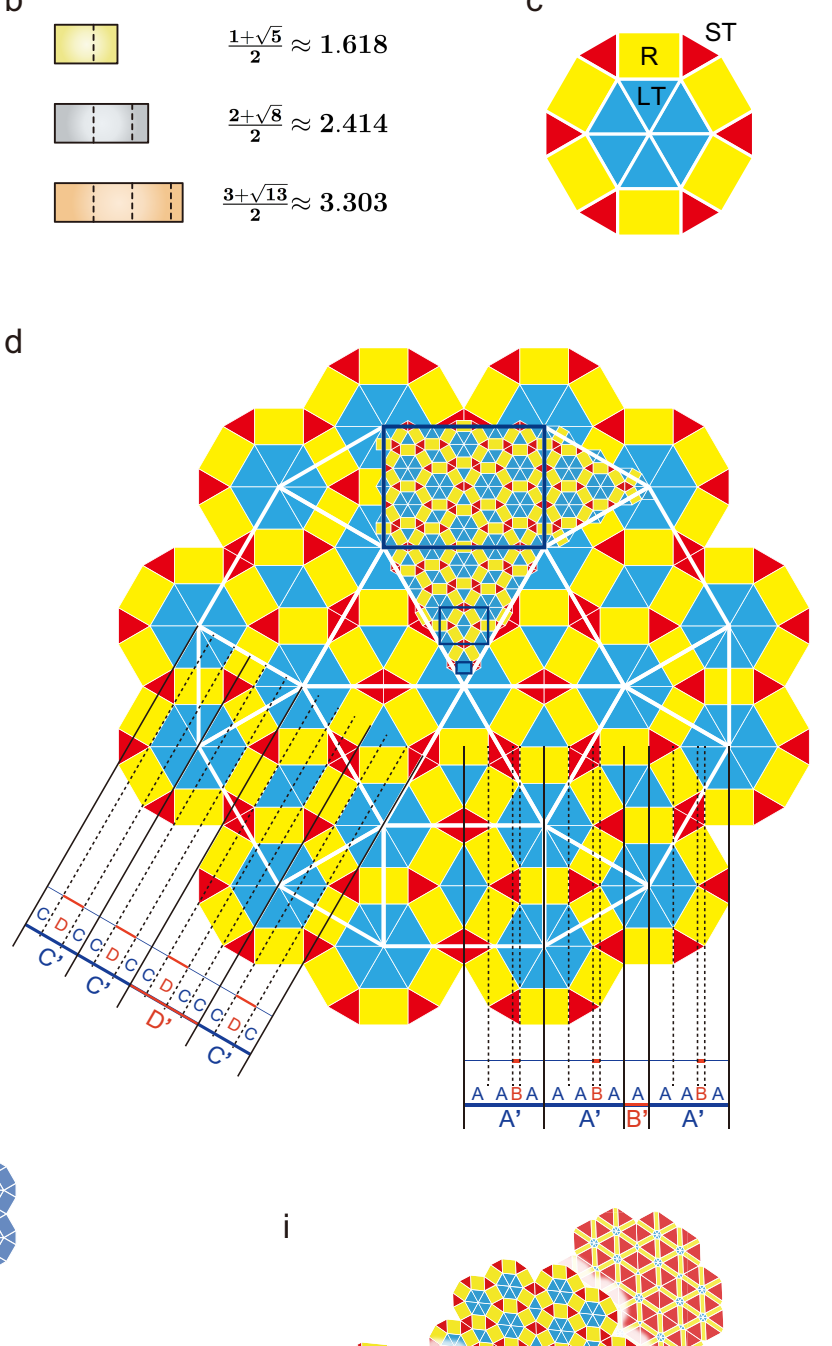

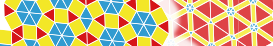

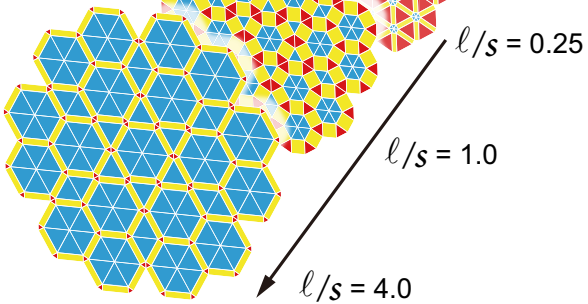


Figure 1. Bronze tiling. a, Subdivision rules: Penrose tiles; Ammann-Beenker tiles; bronzemean tiles consisting of small (ST) and large triangles (LT) and rectangles (R) with the ideal length ratio $\ell / s=(\sqrt{3}+\sqrt{39}) / 6 \approx 1.330$. Indicated are the two lengths characterising the bronze tiles as well as the single lengths characterising the Penrose and the Ammann-Beenker tiles. b, Golden-, silver-, and bronze-mean rectangles with the corresponding aspect ratios. c, The fundamental dodecagon. d, Second-generation pattern obtained by putting the fundamental dodecagons at vertices of the first-generation pattern (white outline), the two sets of parallel lines showing vertex spacings. The black-outlined rectangles in the superimposed portion of the third-generation pattern are self-similar. e, Second-generation ST, LT, and R tiles constructed from identical triangles and squares, with edges decorated so as to enforce subdivision rules from panel a. f, Third-generation $\mathrm{R}$ tile showing bronze-mean sequence in subdivision of long and short edges. $\mathrm{g}$, Second-generation Stampfli tiling showing the two possible orientations of dodecagons, one in light blue and the other in dark blue. h, Second-generation bronze tiling with a single possible orientation of dodecagons. $\mathbf{i}$, Generalised non-self-similar tilings at three length ratios $\ell / s . \mathbf{j}, \mathbf{k}, \mathbf{l}$, Three other patterns based on ST, LT, and R tiles. 
a

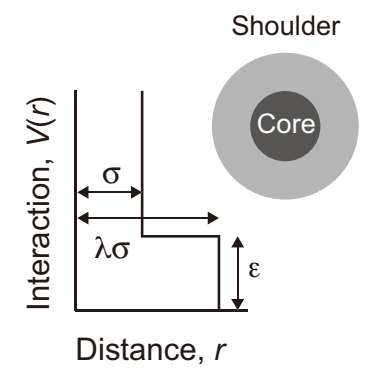

b

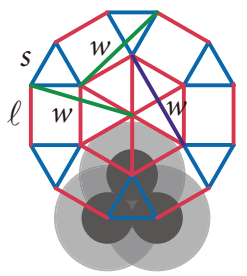

C

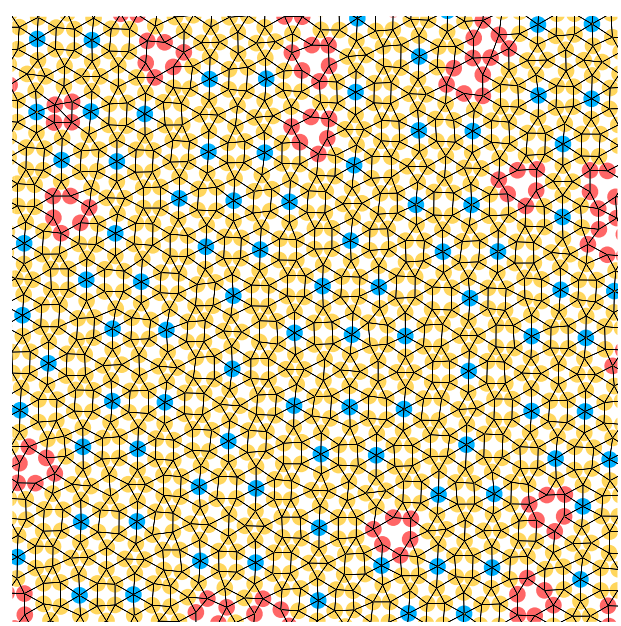

d

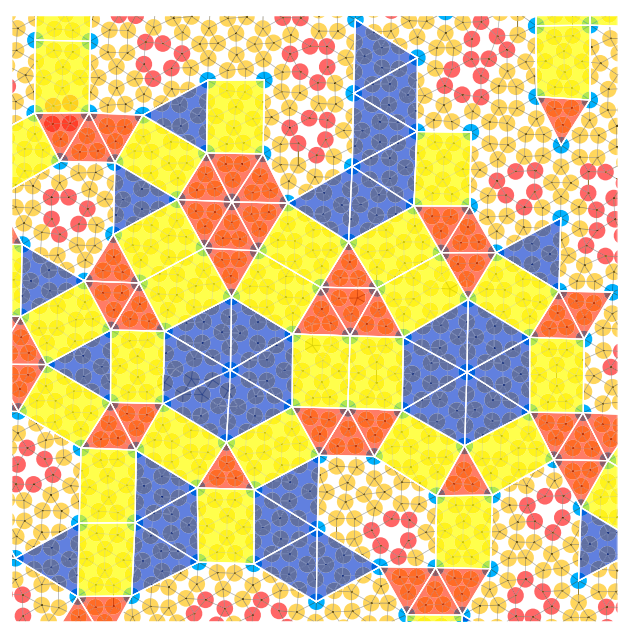

Figure 2. | Hard-core/square-shoulder random bronze quasicrystal. a, Schematic of core-shell particle and HCSS pair interaction. b, Nearest neighbour ( $s$ and $\ell$ ) and next-nearest neighbour $(w)$ distances drawn in the dodecagonal and rugby-ball local motifs. c, Snapshot of a quarter of a 4000-particle QC with shoulder-to-core ratio $\lambda=2.18$, packing fraction $\eta=0.595$, and reduced temperature $k_{\mathrm{B}} T / \varepsilon=0.25$. Cores of particles at the $3^{6}$ and $3^{2} .4 .3 .4$ vertices are coloured blue and yellow, respectively; shoulders are not shown. Cores of particles around shield-shaped defects are coloured red. d, Snapshot from panel $\mathbf{c}$ with highlighted patches of second-generation tiles (red, blue, and yellow) which form several complete and incomplete dodecagons. e, Averaged Fourier transform obtained in simulations (left) with superimposed transform of BMQC generated by higher-dimensional method (right). Spot area is proportional to intensity. Also shown are self-similar patterns of hexagons and rectangles; circles are guides to the eye. 


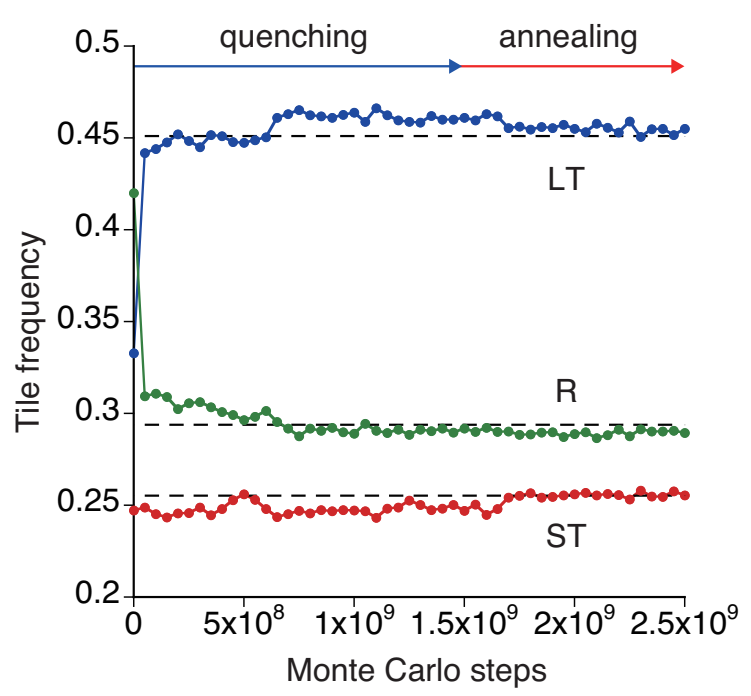

Figure 3. | Tile frequencies. Relative frequencies of ST, LT, and R tiles during simulation of the $\lambda=2.18$ HCSS ensemble. Frequencies were computed by excluding the shield-shaped defects. Also indicated are the ideal frequencies (dashed lines). 


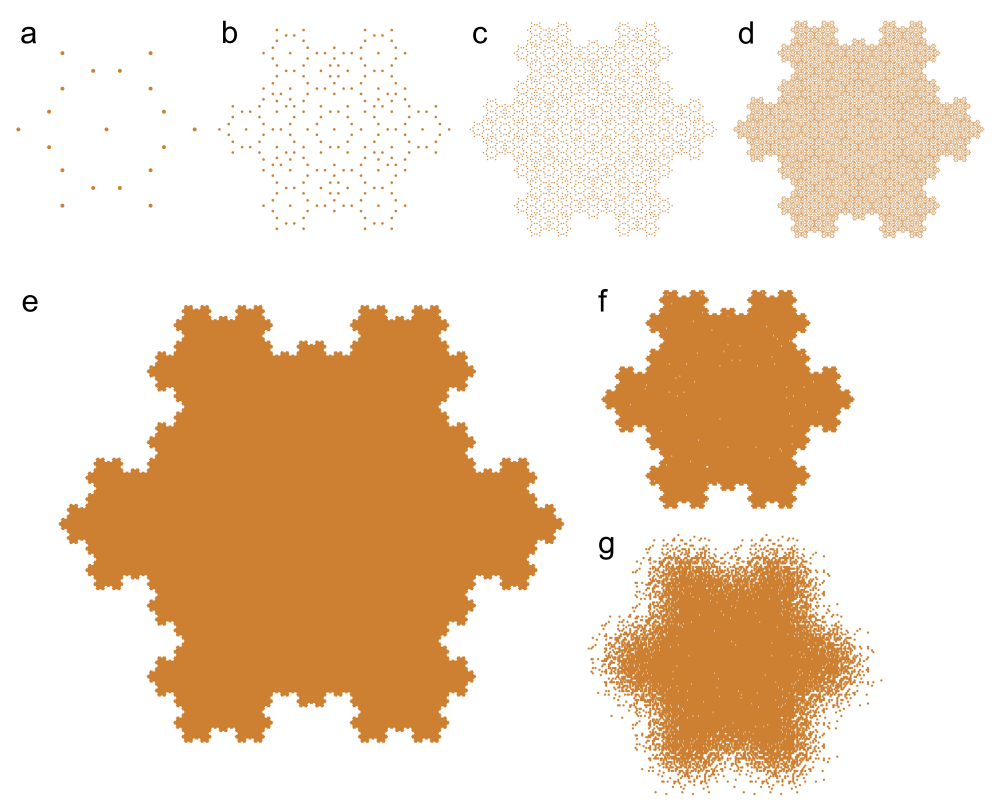

Figure 4. Higher-dimensional analysis. a-e, Projection windows obtained by mapping firstto fifth-generation bronze tilings to the perpendicular space, approaching the Koch-like snowflake fractal shape. The numbers of points are 19, 235, 2677, 29605, and 32439, respectively. f, g, Particle positions in perpendicular space obtained in simulations of $N=14389$ HCSS particles with shoulder-to-core ratio $\lambda=2.18$ by thermalising a rectangle approximant (Supplementary Information IVB) at reduced temperatures $k_{\mathrm{B}} T / \varepsilon=0.2(\mathbf{f})$ and $0.25(\mathbf{g})$. 


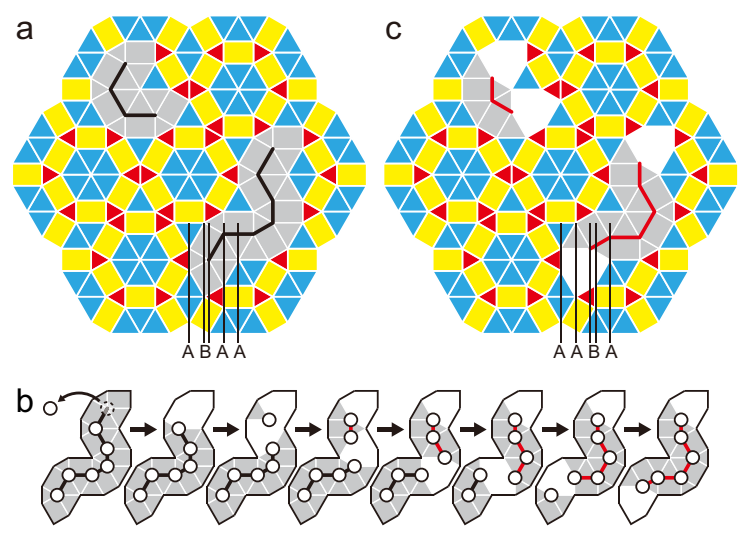

Figure 5. Phason defects. a, A portion of a bronze tiling before the two grey regions undergo zipper moves; their backbones are drawn by bold lines. b, Rearrangement of the larger grey region from panel a illustrating the creation of shield-shaped holes. Vertices along the backbone are decorated by circles for clarity. c, Tiling after zipper rearrangements of the grey regions. The vertical lines in the bottom-centre parts of panels a and $\mathbf{c}$ show a one-dimensional phason flip of lattice planes $\{\mathrm{ABAA}\} \leftrightarrow\{\mathrm{AABA}\}$. 


\title{
Supplementary Information: Bronze-mean hexagonal quasicrystal
}

\author{
Tomonari Dotera, ${ }^{1}$ Shinichi Bekku, ${ }^{1}$ and Primož Ziherl ${ }^{2,3}$ \\ ${ }^{1}$ Department of Physics, Kindai University, 3-4-1 Kowakae Higashi-Osaka 577-8502, Japan \\ ${ }^{2}$ Faculty of Mathematics and Physics, University of Ljubljana, Jadranska 19, SI-1000 Ljubljana, Slovenia \\ ${ }^{3}$ Jožef Stefan Institute, Jamova 39, SI-1000 Ljubljana, Slovenia
}

\section{CONTENTS}

I. Ammann-Beenker tiling

II. Geometry of bronze-mean quasicrystal

III. Simulations of BMQC

IV. BMQC approximants

A. Basic approximants

B. Rectangle approximants

C. Stability of basic approximants

V. Higher-dimensional description
A. Projection matrices
B. Perpendicular space fluctuations
C. Fourier transform
D. Comparison with simulations

VI. Stability of ideal BMQC in view of phason fluctuations

References
1

1

3

5

5

5

6

7

7

9

9

10

10

11

\section{AMMANN-BEENKER TILING}

The square and $45^{\circ}-135^{\circ}$ rhombus tiles in Fig. 1a of the main text give rise to the Ammann-Beenker tiling with 8-fold rotational symmetry shown in Fig. S1, which is the silver-mean analog of the well-known golden-mean Penrose tiling.

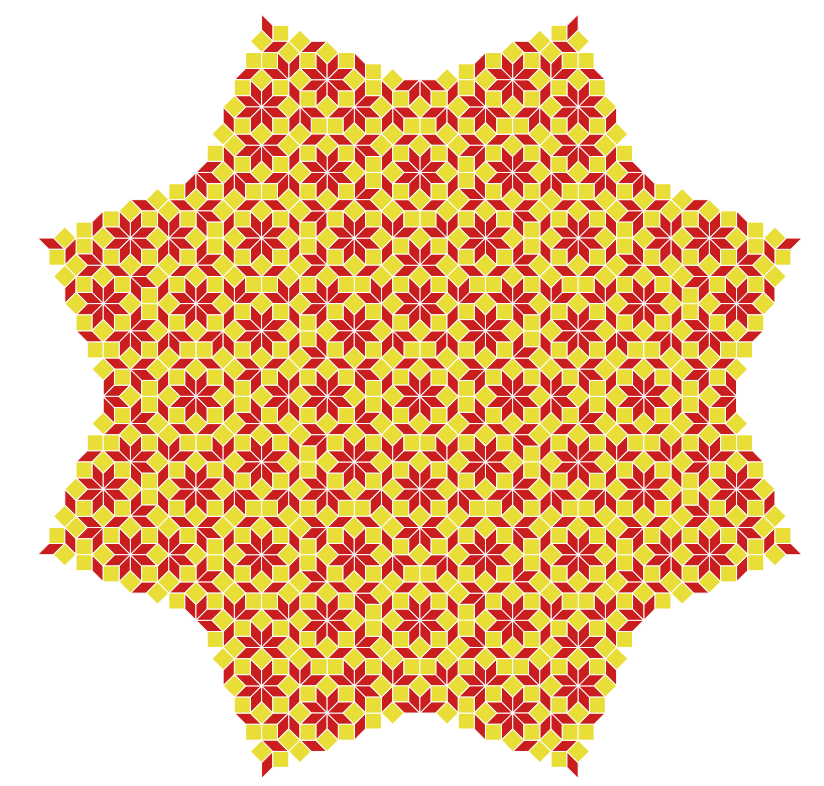

Fig. S1. Ammann-Beenker tiling based on square and $45^{\circ}-$ $135^{\circ}$ rhombus tiles.

\section{GEOMETRY OF BRONZE-MEAN QUASICRYSTAL}

To generate self-similar bronze-mean quasicrystal (BMQC), we begin with a fundamental dodecagon which contains the first-generation vertices (Fig. 1c of the main text). The next-generation tiling is obtained by magnifying the previous-generation tiling by the bronze mean and then by placing the fundamental dodecagons at the vertices of the previous-generation tiling (Supplementary Movie 1). This procedure is called inflation. The vertices of the first- to fourth-generation tilings obtained by three consecutive inflations are shown in Fig. S2: The numbers of points are 19, 235, 2677, and 29605, respectively.

In Fig. S2, we find that the inflated tilings are exactly self-similar in the sense that the next-generation tiling 


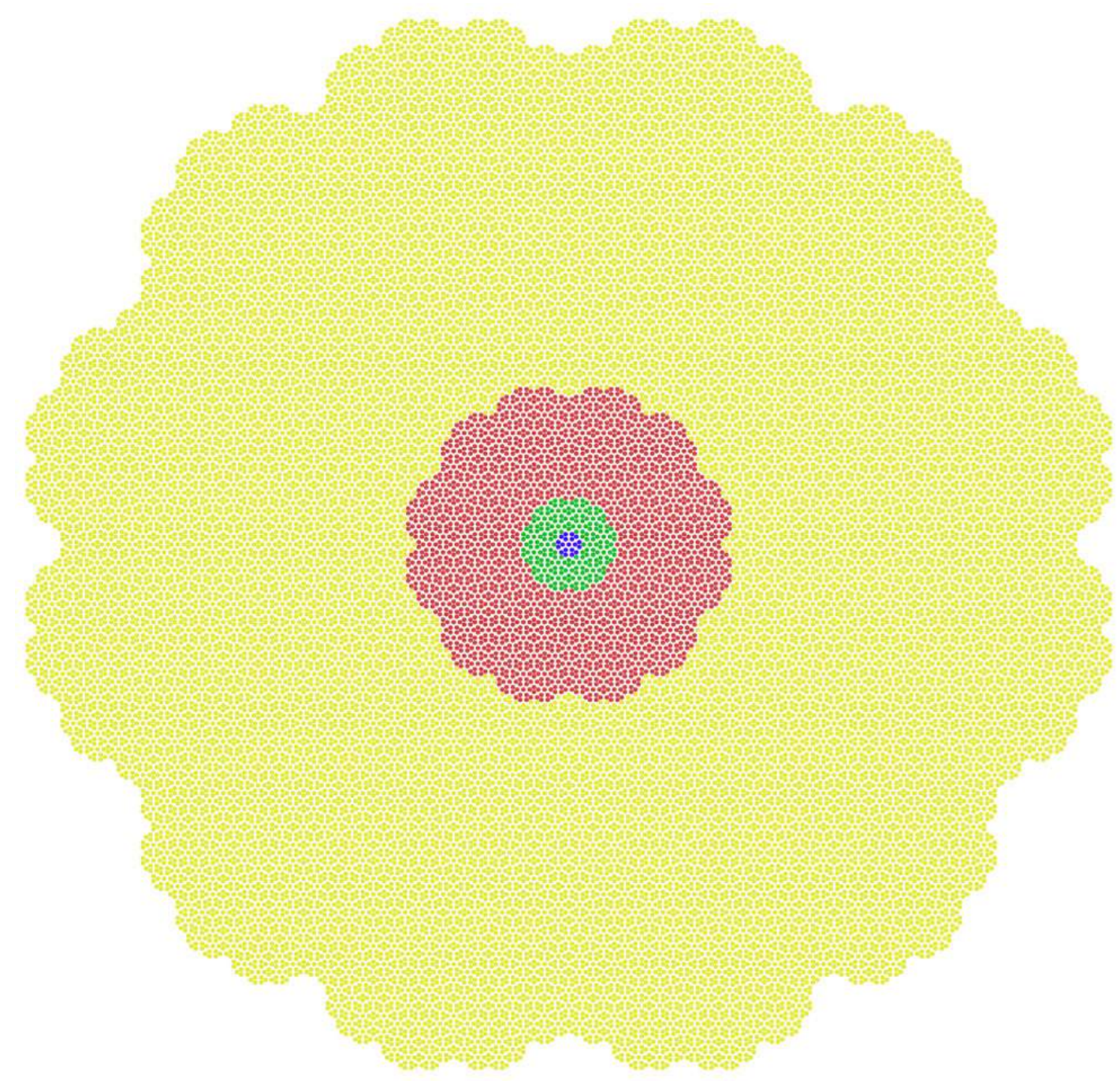

Fig. S2. The first-, second-, third-, and fourth-generation self-similar BMQC constructed by consecutive inflations. In this representation, we plot the vertices of each tiling using coloured dots, the dots of the first, second, third, and fourth generation being blue, green, red, and yellow, respectively; the numbers of points are 19, 235, 2677, and 29605, respectively.

contains the original generation in the centre. With a special length ratio $\ell / s=(\sqrt{3}+\sqrt{39}) / 6 \approx 1.32951$, the rectangular tiles (Fig. 1a of the main text) at every scale are self-similar; this is shown in Fig. 1d of the main text.

The bronze tiling can also be viewed as a covering by cogwheel prototiles. The prototile consists of the dodecagonal pattern (Fig. 1c of the main text) extended by $6 \mathrm{LT}$ tiles arranged around the perimeter and located at the long edges (Fig. S3a). The cogwheel prototile is analogous to the Gummelt decagon devised to reinterpret the Penrose tiling ${ }^{1}$, and the covering is characterised by three types of wheel-wheel overlaps best appreciated when inflating the rectangle: The wheels on the short edge share $2 \mathrm{LT}, 2 \mathrm{ST}$, and $1 \mathrm{R}$ tile, those on the long edge share $2 \mathrm{LT}$ teeth, and those on the diagonal do not overlap (Fig. S3b).

The bronze tiling is fundamentally different from the

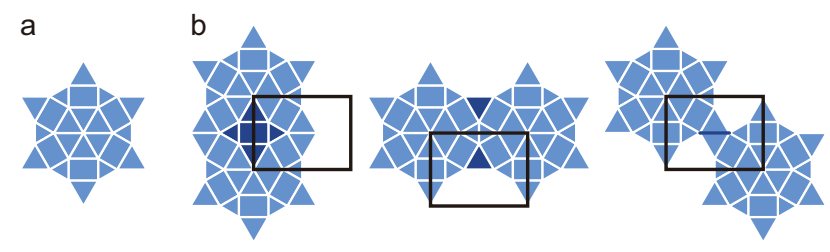

Fig. S3. a, Cogwheel BMQC prototile, b, Three types of the cogwheel overlaps seen in the $\mathrm{R}$ tile; the overlapping portions are highlighted by a dark shade of grey.

Stampfli square-triangle tiling, which is defined by the dodecagonal pattern consisting of 12 triangles and 6 squares (Fig. S4a) and by the triangle and square subdivision rules (two of these rules are shown in Fig. S4b). On application of the subdivision rules, the dodecagon in Fig. 
S4 is transformed into the second-generation dodecagon in Fig. $1 \mathrm{~g}$ of the main text, with the second-generation dodecagons at the perimeter completed for clarity. a

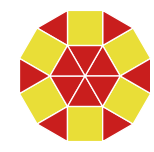

b

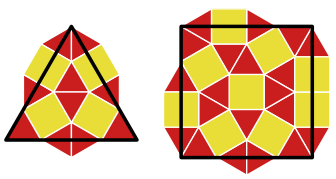

Fig. S4. a, Stampfli dodecagon tessellated into squares and triangles. $\mathbf{b}$, Two examples of subdivision rules for the triangle and the square tiles.

\section{SIMULATIONS OF BMQC}

Our physical model of BMQC is based on a 2D ensemble of two-dimensional core-shell particles with the so-called hard-core/square-shoulder (HCSS) interaction which reads

$$
V(r)= \begin{cases}\infty, & r<\sigma \\ \varepsilon, & \sigma<r<\lambda \sigma . \\ 0, & r>\lambda \sigma\end{cases}
$$

Here $\sigma$ and $\lambda \sigma$ are the core and shoulder diameters, respectively, and $\varepsilon$ is the shoulder height.

Apart from the shoulder-to-core ratio $\lambda$, a key parameter controlling the structure of the HCSS system is the packing fraction defined as the ratio of the area occupied by the particles' hard cores $\mathcal{D}$ in a given tiling and the total area of the tiling $\mathcal{A}$ :

$$
\eta=\frac{\mathcal{D}}{\mathcal{A}}
$$

It is helpful to calculate the packing fractions of the three types of tiles as a function of $\lambda$; the results are listed in Table SI together with $\mathcal{D}$ and $\mathcal{A}$ in units of $\sigma^{2}$. Note that the hard-core area of a particle is $\pi \sigma^{2} / 4$ and that the natural choice for the short length of the tiling $s=\sigma$.

\begin{tabular}{|c|c|c|c|}
\hline & $\mathcal{D} / \sigma^{2}$ & $\mathcal{A} / \sigma^{2}$ & $\eta(\lambda)$ \\
\hline ST & $\pi / 8$ & $\sqrt{3} / 4$ & $\sqrt{3} \pi / 6$ \\
\hline LT & $\pi / 8$ & $\sqrt{3} \lambda^{2} / 12$ & $\sqrt{3} \pi /\left(2 \lambda^{2}\right)$ \\
\hline R & $\pi / 4$ & $\sqrt{3} /(3 \lambda)$ & $\sqrt{3} \pi /(4 \lambda)$ \\
\hline
\end{tabular}

Table S I. Area occupied by particles $(\mathcal{D})$, tile area $(\mathcal{A})$, both in units of $\sigma^{2}$, and packing fraction $\eta$ of the ST, LT, and R tiles as a function of shoulder-to-core ratio $\lambda$.

As discussed in the main text, a physical model of the disordered BMQC is expected in the HCSS ensemble at the shoulder-to-core ratio $\lambda^{*}=(3+\sqrt{33}) / 4 \approx 2.18614$ corresponding to $\ell^{*} / s=(\sqrt{3}+\sqrt{11}) / 4 \approx 1.26217$. In this case, the packing fractions of $\mathrm{ST}, \mathrm{LT}$, and R tiles are $0.906900,0.569278$, and 0.622261 , respectively. Notice that $\ell^{*} / s$ is not equal to the value of the length ratio in the exact self-similar tiling $\ell / s=(\sqrt{3}+\sqrt{39}) / 6 \approx$ 1.32951.

We performed many runs of Monte-Carlo simulations of a two-dimensional HCSS ensemble of $N=4000$ particles in a square box with periodic boundary conditions, starting from randomised states produced at the infinite temperature. A typical run consisted of $1-3 \times 10^{9}$ Monte Carlo (MC) steps as appropriate. In our experience, quenching is necessary to avoid metastable states, and slow annealing at high temperatures, say at $k_{\mathrm{B}} T / \varepsilon=0.25$, is needed to obtain high-quality QCs with small linear phason strains.

We focused on HCSS particles at a shoulder-to-core ratio of 2.18 which is reasonably close to $\lambda^{*}$; the packing fraction was $\eta=0.595$. Figure S5 show the energy per

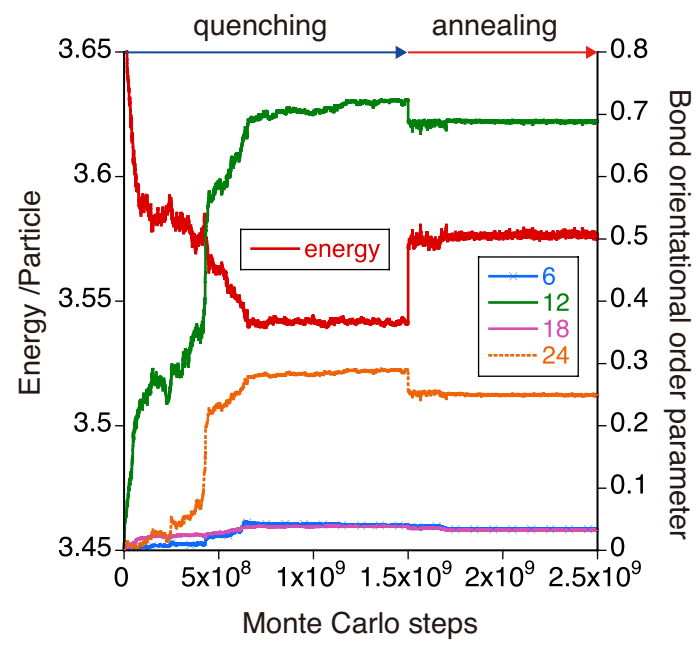

Fig. S5. Energy per particle in the unit of $\varepsilon$ and bondorientational order parameters $\chi_{m}(m=6,12,18$, and 24$)$ vs. Monte Carlo (MC) steps. The system is quenched at a reduced temperature $k_{\mathrm{B}} T / \varepsilon=0.20408$ up to $1.5 \times 10^{9} \mathrm{MC}$ steps and then annealed at a temperature $k_{\mathrm{B}} T / \varepsilon=0.25$.

particle, the bond-orientational order parameters, and the frequencies of tiles for a system initially quenched at a reduced temperature $k_{\mathrm{B}} T / \varepsilon=0.20408$ for $1.5 \times 10^{9}$ $\mathrm{MC}$ steps and then annealed at a higher temperature $k_{\mathrm{B}} T / \varepsilon=0.25$. The energy is almost constant during the second half of the quenching stage (from $7 \times 10^{8}$ to $1.5 \times 10^{9} \mathrm{MC}$ steps) and during the annealing stage from $1.7 \times 10^{9}$ MC step on.

The growth of rotational order during the quenching and annealing stages is seen in Fig. S5 showing bondorientational order parameters $\chi_{m}$ defined as

$$
\chi_{m}=\left\langle\left|\frac{1}{N_{B}} \sum_{\mathbf{r}} \exp \left(i m \theta_{\mathbf{r}}\right)\right|^{2}\right\rangle,
$$

where $\theta_{\mathbf{r}}$ is the angle between an arbitrary axis and a 

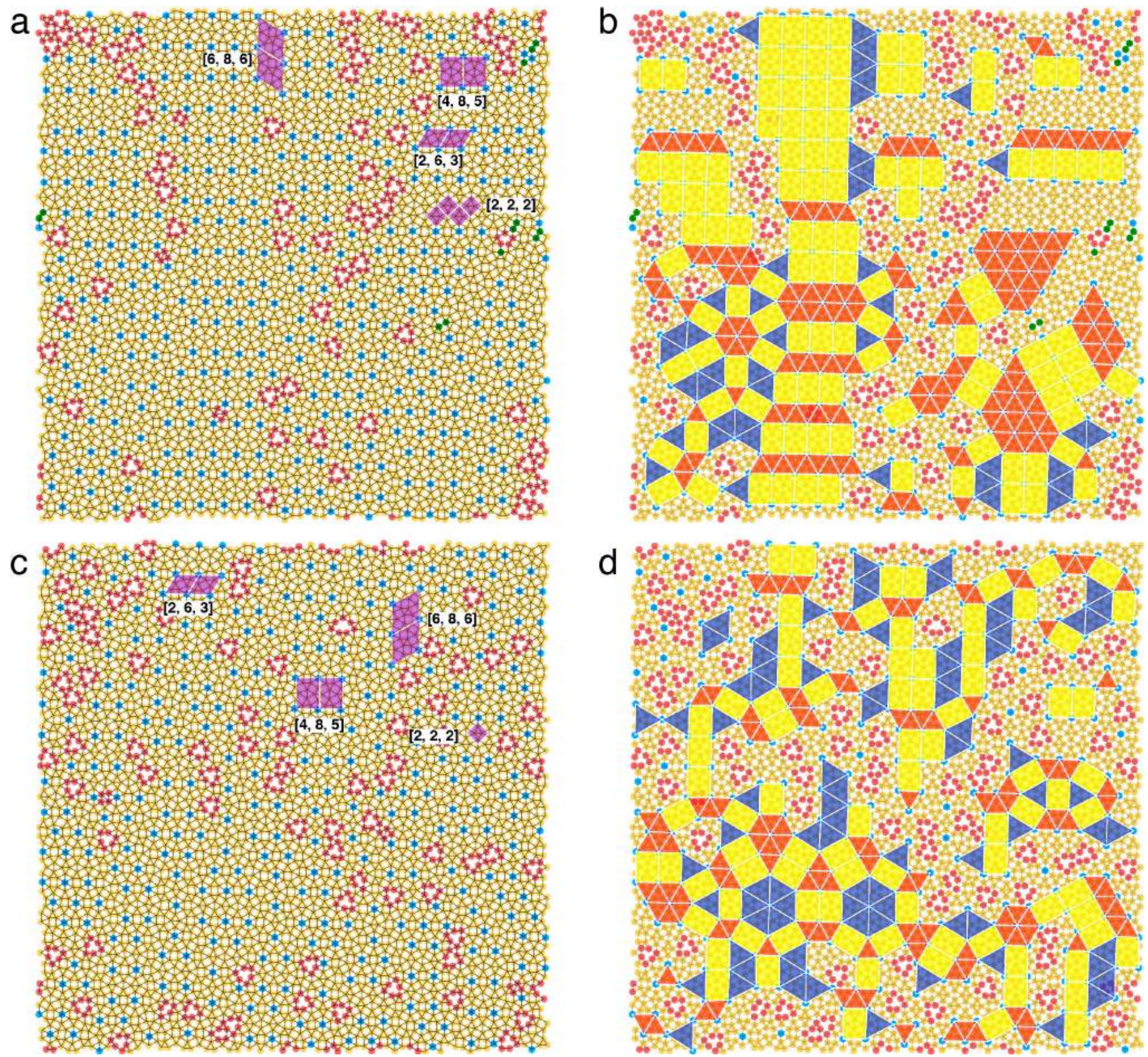

Fig. S6. a, Snapshot of the $\lambda=2.18 \mathrm{HCSS}$ ensemble consisting of $N=4000$ particles quenched at $k_{\mathrm{B}} T / \varepsilon=0.20408$. Sizable patches of $[2,6,3]$ and $[4,8,5]$ basic approximants are readily visible, and representatives of the approximant unit cells are drawn in magenta. b, Snapshot from panel a with highlighted patches of second-generation $[2,6,3]$, [6, 8, 6], and [4, 8, 5] tiles (red, blue, and yellow, respectively). c, Snapshot of the $\lambda=2.18$ HCSS ensemble annealed at $k_{\mathrm{B}} T / \varepsilon=0.25$. The system is much more homogeneous than the quenched state. d, Snapshot from panel c with with highlighted second-generation tiles which form several complete and incomplete dodecagons. Both self-similar and non-self-similar variants of the dodecagon are readily seen.

bond connecting particles separated by no more than $1.5 \sigma$ and located at $\mathbf{r} ; N_{B}$ is the number of the bonds and angle brackets indicate ensemble average. This value provides global orientational ordering for a system. The key feature that we observe is hexagonal symmetry evidenced by the presence of $\chi_{6}$ and $\chi_{18}$, although $\chi_{12}$ and $\chi_{24}$ are quite large.
We also measured the frequencies of three BMQC tiles (ST, LT, and R). As witnessed by Fig. 3 of the main text, these frequencies approach the ideal values described in the main text. Note that in Fig. 3 of the main text the defects, most of which are shield-shaped, are excluded from the analysis and we only compare the frequencies of the ST, LT, and R tiles. As shown in Table SIII, 
these frequencies are different from those of the basic approximants, which leads us to conclude that our tiling is a random version of the BMQC.

Snapshots of a quenched state at $1.485 \times 10^{9} \mathrm{MC}$ steps are shown in Fig. S6a \& b, and those of the annealed state at a higher temperature $k_{\mathrm{B}} T / \varepsilon=0.25$ are shown in Fig. S6c \& d. In this representation, centres of secondgeneration dodecagons from Fig. 1c of the main text are drawn by blue circles and all other sites consistent with the canonical local structure based ST, LT, R forming dodecagons and rugby-ball motifs (Fig. 2b of the main text) are drawn by yellow circles. Particles forming shieldlike and other structural defects associated with phason strain are drawn by red circles and a few other defect sites are coloured green.

In the quenched state (Fig. S6a), the system readily forms several well-ordered patches of second-generation $[2,6,3]$ and $[4,8,5]$ approximants; $[6,8,6]$ and $[2,2$, $2]$ approximants are also seen. A few representatives of unit cells of these four approximants are drawn in magenta and in Fig. S6b the snapshot is replotted with all second-generation ST, LT, and R tiles highlighted in red, blue, and yellow, respectively. The R-type $[4,8,5]$ grains are readily visible; the ST-type $[2,6,3]$ approximant appears both at the boundaries of the $[4,5,8]$ grains as well as in the form of standalone grains. On the other hand, large grains of LT-type $[6,8,6]$ approximant are absent. The bottom-left part of the panel contains one complete non-self-similar second-generation low-density dodecagon (Fig. $1 \mathrm{j}$ of the main text), and a few fragments of this pattern are also visible elsewhere in the snapshot.

After annealing at a higher temperature $k_{\mathrm{B}} T / \varepsilon=0.25$ (Fig. S6c at $2.555 \times 10^{9} \mathrm{MC}$ steps), the second-generation approximant grains are almost completely dispersed and several generally incomplete first-generation dodecagons appear instead. These structures can be recognized in Fig. S6d where second-generation ST, LT, and R tiles are indicated using the same color code as in Fig. S6b. Two complete self-similar low-density dodecagons (Fig. 1c of the main text) can be seen in the bottom-left part of the panel, and several incomplete second-generation dodecagons are scattered across all of the domain. Both self-similar and non-self-similar variants of the dodecagon are present.

\section{BMQC APPROXIMANTS}

\section{A. Basic approximants}

Based on the characteristic types of overlapping and non-overlapping packings of the fundamental dodecagonal and the rugby-ball motif in Fig. 1c and Fig. 2b of the main text, we can construct several $2 \mathrm{D}$ lattices which include certain local structural features of BMQCs. Five such approximants, referred to as the basic approximants, are shown in Fig. S7. The appoximants are la-
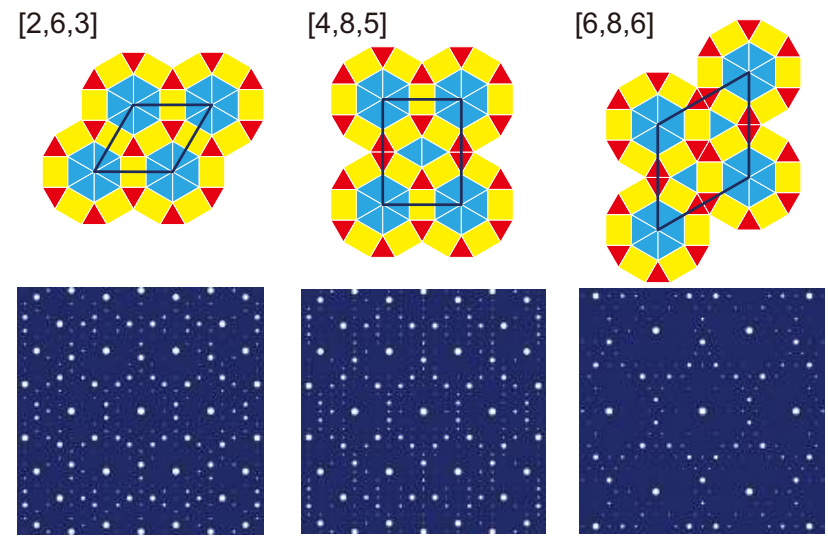

$[2,2,2]$

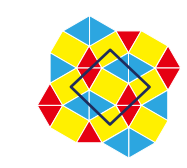

$[8,6,6]$
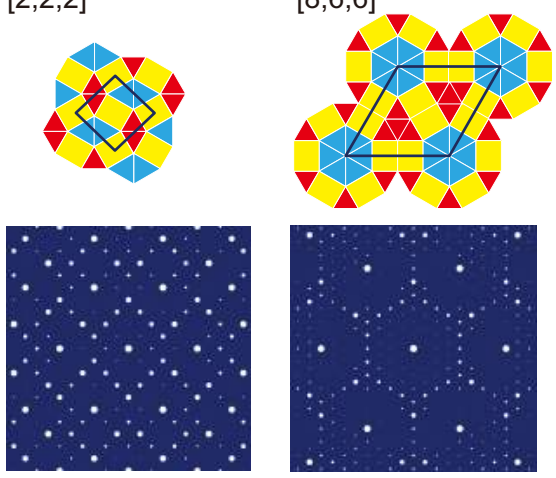

Fig. S7. Basic approximants $[2,6,3],[4,8,5],[6,8,6]$, $[2,2,2]$, and $[8,6,6]$, the notation $[k, l, m]$ referring to an approximant consisting of $k \mathrm{ST}, l \mathrm{LT}$, and $m \mathrm{R}$ tiles. Unit cells are outlined in each panel, and Fourier transforms are presented below each structure.

belled by triplets $[k, l, m]$ where $k, l$, and $m$ are the numbers of ST, LT, and R tiles in the unit cell. Also shown are the Fourier transform images of the approximants.

The approximants $[2,6,3],[4,8,5]$, and $[6,8,6]$ are nothing but the subdivision rules of the BMQC. Except the $[8,6,6]$ approximant, all patterns shown here may also be viewed as coverings of the dodecagonal and the rugby ball patterns in Fig. $2 \mathrm{~b}$ of the main text, and are thus energetically favourable in case of the pair potential described in Sec. III. We note that the $[2,2,2]$ approximant is found in an oxide quasicrystal approximant (Ref. 28 in the main text).

\section{B. Rectangle approximants}

There are several ways to construct a sequence of approximants which tend to ideal quasicrystals. Here we illustrate a series of rectangle approximants. We start with a single rectangle as the first generation and apply inflation, in which the number of polygons grows as described by Eq. (4) of the main text. Table SII lists the numbers of vertices and the number of tiles in a unit cell 
as well as lattice constants for the $n$-th generation. We use these approximants in Sec. VI to study the stability of BMQC.
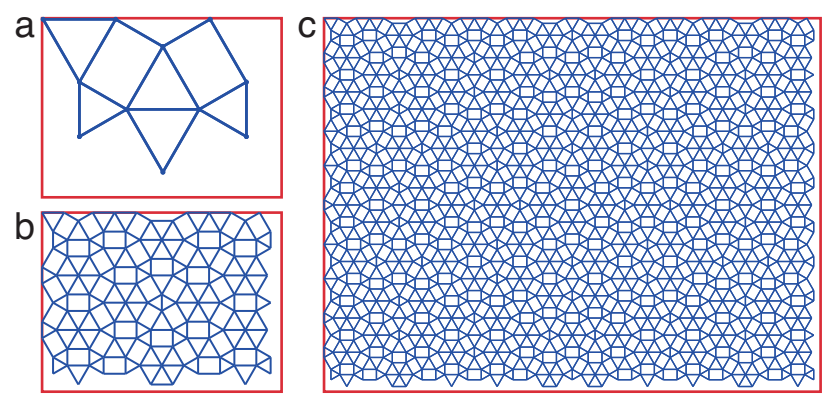

Fig. S8. Rectangle approximants: a, second generation, b, third generation, and $\mathbf{c}$, fourth generation, respectively. Unit cells are outlined in red. The second generation is the $[4,8$, 5] basic approximant.

\begin{tabular}{|r|r|r|r|r|r|r|}
\hline$n$ & $N_{n}$ & $S T_{n}$ & $L T_{n}$ & $R_{n}$ & $\boldsymbol{L}_{x}$ & $\boldsymbol{L}_{y}$ \\
\hline 1 & 1 & 0 & 0 & 1 & $(0,2,0,-1)$ & $(-1,0,2,0)$ \\
2 & 11 & 4 & 8 & 5 & $(3,4,0,-2)$ & $(-1,0,2,3)$ \\
3 & 121 & 48 & 84 & 55 & $(9,14,0,-7)$ & $(-4,0,8,9)$ \\
4 & 1319 & 599 & 920 & 520 & $(30,46,0,-23)$ & $(-13,0,26,30)$ \\
5 & 14389 & 5676 & 10032 & 6535 & $(99,152,0,-76)$ & $(-43,0,86,99)$ \\
\hline
\end{tabular}

Table S II. Rectangle approximants: Numbers of vertices $N_{n}$, small triangles $S T_{n}$, large triangles $L T_{n}$, and rectangles $R_{n}$ in a unit cell, and lattice constants given by indices $\left(n_{1}, n_{2}, n_{3}, n_{4}\right)$ in the form of $\boldsymbol{L}_{\alpha}^{\|}=\sum_{i=1}^{4} n_{i} \boldsymbol{e}_{i}^{\|}$for rectangle approximants of the $n$-th generation; $\alpha$ stands for $x$ and $y$. Basis vectors $\boldsymbol{e}_{i}^{\|}$are shown in Fig. S10a.

\section{Stability of basic approximants}

In order to better understand the structural motifs observed in simulations, it is instructive to analyse the stability of the approximants at $T=0$. To this end, we evaluate their enthalpy $H=E+P V$ where $E$ and $V$ are the overlap energy and the area per particle, and $P$ is pressure. Below we first calculate the packing fraction of the approximants and their overlap energy and then construct the phase diagram.

Before doing so, let us first consider the self-similar BMQC itself (Fig. 1a of the main text). The average core area $\mathcal{D}_{\mathrm{BMQC}}$ and the average total area $\mathcal{A}_{\mathrm{BMQC}}$ in a tiling of $N$ tiles are given by

$$
\begin{aligned}
\frac{\mathcal{D}_{\mathrm{BMQC}}}{N} & =\frac{\pi \sigma^{2}}{8} n_{\mathrm{ST}}+\frac{\pi \sigma^{2}}{8} n_{\mathrm{LT}}+\frac{\pi \sigma^{2}}{4} n_{\mathrm{R}} \\
& =\frac{\pi \sigma^{2}}{172}(17+3 \sqrt{13}),
\end{aligned}
$$

and

$$
\frac{\mathcal{A}_{\mathrm{BMQC}}}{N}=\frac{\sqrt{3}}{4} s^{2} n_{\mathrm{ST}}+\frac{\sqrt{3}}{4} \ell^{2} n_{\mathrm{LT}}+\ell s n_{\mathrm{R}},
$$

respectively. Here $n_{\alpha}$ are the ideal tile frequencies:

$$
\begin{aligned}
& n_{\mathrm{ST}}=\frac{29-5 \sqrt{13}}{43} \approx 0.255168 \\
& n_{\mathrm{LT}}=\frac{23-\sqrt{13}}{43} \approx 0.451034, \\
& n_{\mathrm{R}}=\frac{3(-3+2 \sqrt{13})}{43} \approx 0.293798 .
\end{aligned}
$$

The packing fraction of the BMQC at the shoulder-tocore ratio of $\lambda^{*}$ is $\eta_{\mathrm{B}}^{*}=\mathcal{D}_{\mathrm{B}} / \mathcal{A}_{\mathrm{B}}=0.641146$.

Now we calculate the frequencies of the ST, LT, and $\mathrm{R}$ tiles in the basic approximants in Fig. S7 along with the corresponding packing fractions. The area occupied by the particles' hard cores within the unit cell $\mathcal{D}_{[k, l, m]}$ and the area of the unit cell itself $\mathcal{A}_{[k, l, m]}$ are given by

$$
\mathcal{D}_{[k, l, m]}=\frac{\pi \sigma^{2}}{8} k+\frac{\pi \sigma^{2}}{8} l+\frac{\pi \sigma^{2}}{4} m
$$

and

$$
\mathcal{A}_{[k, l, m]}=\frac{\sqrt{3}}{4} s^{2} k+\frac{\sqrt{3}}{4} \ell^{2} l+\ell s m,
$$

respectively. Tile frequencies and packing fractions $\eta^{*}$ at shoulder-to-core ratio of $\lambda^{*}$ are summarised in Table SIII; also included are the data for the BMQC. For comparison, packing fractions $\eta$ for the exact self-similar tiling are listed as well. The $[2,6,3]$ approximant has the lowest density, whereas the $[8,6,6]$ approximant has the highest density among the approximants considered here.

\begin{tabular}{|c|c|c|c|c|c|}
\hline & $n_{\mathrm{ST}}$ & $n_{\mathrm{LT}}$ & $n_{\mathrm{R}}$ & $\eta^{*}$ & $\eta$ \\
\hline$[2,6,3]$ & 0.18182 & 0.54545 & 0.27273 & 0.62536 & 0.58197 \\
\hline$[4,8,5]$ & 0.23529 & 0.47059 & 0.29412 & 0.63705 & 0.59571 \\
\hline BMQC & 0.25517 & 0.45103 & 0.29380 & 0.64115 & 0.60034 \\
\hline$[6,8,6]$ & 0.30000 & 0.40000 & 0.30000 & 0.65076 & 0.61145 \\
\hline$[2,2,2]$ & 0.33333 & 0.33333 & 0.33333 & 0.65861 & 0.62138 \\
\hline$[8,6,6]$ & 0.40000 & 0.30000 & 0.30000 & 0.67278 & 0.63680 \\
\hline
\end{tabular}

Table S III. Frequencies of small triangles $\left(n_{\mathrm{ST}}\right)$, large triangles $\left(n_{\mathrm{LT}}\right)$ and rectangles $\left(n_{\mathrm{R}}\right)$, and the packing fraction $\eta^{*}$ and $\eta$ of the $[2,6,3],[4,8,5],[2,2,2],[8,6,6]$ and $[6,8,6]$ approximants at shoulder-to-core ratio of $\lambda^{*}$ and at the special length ratio $\ell / s=(\sqrt{3}+\sqrt{39}) / 6 \approx 1.32951$, respectively. Also included are data for the bronze-mean quasicrystal (BMQC).

The average overlap energy per particle in the approximants increases with density as expected, and for shoulder-to-core ratio of $\lambda^{*}$ the overlap energies are listed in Table SIV. Evidently, the overlap energy of the $[8,6$, 


\begin{tabular}{|c|c|c|c|}
\hline & $E / \varepsilon$ & $N$ & $e$ \\
\hline$[2,6,3]$ & 24 & 7 & 3.42857 \\
\hline$[4,8,5]$ & 39 & 11 & 3.54545 \\
\hline$[6,8,6]$ & 48 & 13 & 3.69231 \\
\hline$[2,2,2]$ & 15 & 4 & 3.75000 \\
\hline$[8,6,6]$ & 57 & 13 & 4.38462 \\
\hline
\end{tabular}

Table S IV. Energies of $[2,6,3],[4,8,5],[2,2,2],[6,6,6]$, and $[6,8,6]$ approximants: Listed are the total overlap energy per unit cell in units of shoulder height $E / \varepsilon$, the number of particles per unit cell $N$, and the average reduced energy per particle $e=E /(N \varepsilon)$.

6] approximant is much higher than in other approximants, which is consistent with the fact that it has not been observed in simulations.

At $T=0$ reduced enthalpy per vertex $h=H / N$ is given by

$$
h=e+p v,
$$

where $e$ is the average energy per particle in units of $\varepsilon$, $v=\pi /(4 \eta)$ is the area per particle in units of $\sigma^{2}$, and $p$ is pressure in units of $\varepsilon / \sigma^{2}$.

The reduced enthalpies of the basic approximants in Fig. S7 at shoulder-to-core ratio of $\lambda^{*}$ are plotted in Fig. S9. Except for the $[8,6,6]$ approximant, their enthalpies

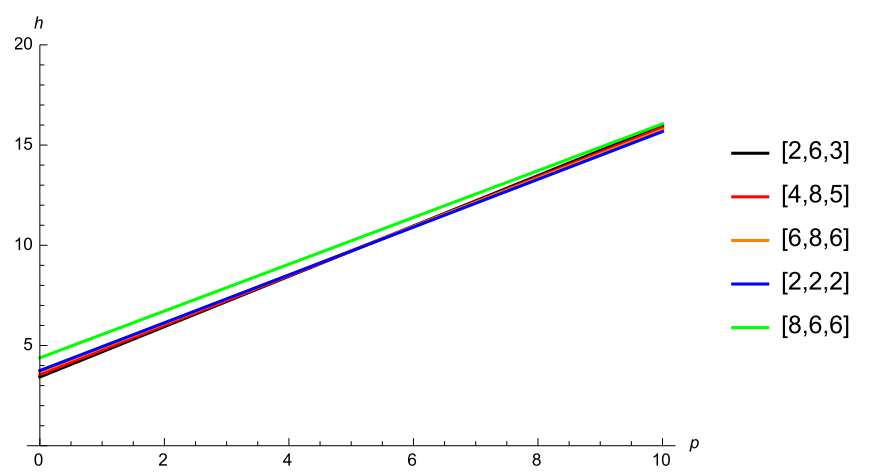

Fig. S9. Reduced enthalpies of the $[2,6,3]$, $[4,8,5]$, [6, 8, 6], $[2,2,2]$, and $[8,6,6]$ basic approximants at shoulder-to-core ratio of $\lambda^{*}$ vs. pressure.

are very close to each other across a broad range of reduced pressures. Up to $p=5.06819$ the lowest-enthalpy state is the $[2,6,3]$ approximant and at $p>5.06819$ it is the $[2,2,2]$ approximant. At $p=5.06819$, the enthalpy of the $[4,8,5]$ approximant is the same as that of the $[2,6,3]$ and the $[2,2,2]$ approximants (i.e., $\left.h_{[4,8,5]}=h_{[2,6,3]}=h_{[2,2,2]}\right)$; given that the $[4,8,5]$ approximant combines overlapping dodecagons of the $[2,6$, $3]$ approximant with the rugby-ball motif of the $[2,2,2]$ approximant, this is expected. At this pressure, $h_{[6,8,6]}$ is larger than $h_{[4,8,5]}=h_{[2,6,3]}=h_{[2,2,2]}$ by no more than $0.156 \%$. The very small differences in enthalpies of the $[2$,
$6,3],[4,8,5],[6,8,6]$, and $[2,2,2]$ approximants is consistent with their presence in simulations; the enthalpybased argument also explains the absence of the $[8,6,6]$ approximant.

\section{HIGHER-DIMENSIONAL DESCRIPTION}

\section{A. Projection matrices}

Here we outline the main steps of the construction of the bronze tiling by projection of a higher-dimensional hyperlattice onto the physical space. We first derive projection operators producing the self-similar hexagonal quasicrystal using the higher-dimensional analysis ${ }^{2,3}$. In this Section we use $s$ as the unit length so as to make the notation more concise; thus here $\ell$ is the dimensionless ratio of the long and short lengthscale. Let us denote the orthogonal basis vectors generating the six-dimensional hyperlattice by $\boldsymbol{e}_{i}, i=1, \cdots, 6$, where $\boldsymbol{e}_{i} \cdot \boldsymbol{e}_{j}=\delta_{i j}$, and $\delta_{i j}$ is the Kronecker's delta. The quasi-twelvefold symmetry corresponds to cyclic permutations

$$
\left(\begin{array}{l}
1 \\
0 \\
0 \\
0 \\
0 \\
0
\end{array}\right) \rightarrow\left(\begin{array}{l}
0 \\
\ell \\
0 \\
0 \\
0 \\
0
\end{array}\right) \rightarrow \cdots \rightarrow\left(\begin{array}{l}
0 \\
0 \\
0 \\
0 \\
0 \\
\ell
\end{array}\right) \rightarrow\left(\begin{array}{c}
-1 \\
0 \\
0 \\
0 \\
0 \\
0
\end{array}\right) .
$$

Thus the rotation matrix is given by

$$
T=\left(\begin{array}{cccccc}
0 & 1 / \ell & 0 & 0 & 0 & 0 \\
0 & 0 & \ell & 0 & 0 & 0 \\
0 & 0 & 0 & 1 / \ell & 0 & 0 \\
0 & 0 & 0 & 0 & \ell & 0 \\
0 & 0 & 0 & 0 & 0 & 1 / \ell \\
-\ell & 0 & 0 & 0 & 0 & 0
\end{array}\right)
$$

The eigenvalues of the $T$ matrix are $i,-i, z,-z, z^{5},-z^{5}$, where $z=\exp (i \pi / 6)$. The eigenvector associated with $z$ is $\left(1, \ell z, z^{2}, \ell z^{3}, z^{4}, \ell z^{5}\right)$. The real and imaginary parts of this vector

$$
\boldsymbol{a}_{1}^{\|}=\sqrt{\frac{1}{6\left(\ell^{2}+1\right)}}(2, \sqrt{3} \ell, 1,0,-1,-\sqrt{3} \ell)
$$

and

$$
\boldsymbol{a}_{2}^{\|}=\sqrt{\frac{1}{6\left(\ell^{2}+1\right)}}(0, \ell, \sqrt{3}, 2 \ell, \sqrt{3}, \ell)
$$

respectively, span an invariant subspace corresponding to the physical space. Then the projection operator onto the physical space is given by

$$
\begin{gathered}
P_{j k}=\sum_{i=1}^{2}\left(\boldsymbol{a}_{i}^{\|} \cdot \boldsymbol{e}_{j}\right)\left(\boldsymbol{a}_{i}^{\|} \cdot \boldsymbol{e}_{k}\right) \\
=\frac{1}{3\left(\ell^{2}+1\right)}\left(\begin{array}{rrrrrr}
2 & \sqrt{3} \ell & 1 & 0 & -1 & -\sqrt{3} \ell \\
\sqrt{3} \ell & 2 \ell^{2} & \sqrt{3} \ell & \ell^{2} & 0 & -\ell^{2} \\
1 & \sqrt{3} \ell & 2 & \sqrt{3} \ell & 1 & 0 \\
0 & \ell^{2} & \sqrt{3} \ell & 2 \ell^{2} & \sqrt{3} \ell & \ell^{2} \\
-1 & 0 & 1 & \sqrt{3} \ell & 2 & \sqrt{3} \ell \\
-\sqrt{3} \ell & -\ell^{2} & 0 & \ell^{2} & \sqrt{3} \ell & 2 \ell^{2}
\end{array}\right),
\end{gathered}
$$


where $P^{2}=P$ and $P e_{k}=e_{k}^{\|}$. Then one finds

$$
\left|\boldsymbol{e}_{k}^{\|}\right|=\sqrt{\frac{2}{3\left(\ell^{2}+1\right)}} \quad \text { for } k=1,3,5
$$

and

$$
\left|\boldsymbol{e}_{k}^{\|}\right|=\ell \sqrt{\frac{2}{3\left(\ell^{2}+1\right)}} \text { for } k=2,4,6 \text {. }
$$

These projected basis vectors are shown in Fig. S10a.
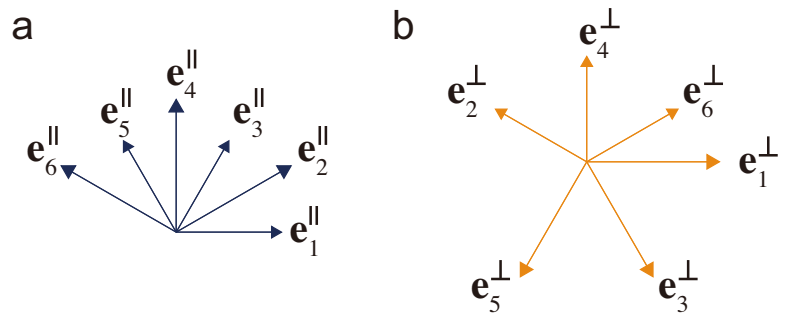

Fig. S10. a, Projected basis vectors in the physical space $\boldsymbol{e}_{i}^{\|}$. $\mathbf{b}$, Projected basis vectors in the perpendicular space $\boldsymbol{e}_{i}^{\perp}$.

The perpendicular space is spanned by

$$
\boldsymbol{a}_{1}^{\perp}=\sqrt{\frac{1}{6\left(\ell^{2}+1\right)}}(2 \ell,-\sqrt{3}, \ell, 0,-\ell, \sqrt{3})
$$

and

$$
\boldsymbol{a}_{2}^{\perp}=\sqrt{\frac{1}{6\left(\ell^{2}+1\right)}}(0,1,-\sqrt{3} \ell, 2,-\sqrt{3} \ell, 1) .
$$

producing another invariant subspace corresponding to the perpendicular space. The projection operator onto the perpendicular space reads

$$
\begin{gathered}
Q_{j k}=\sum_{i=1}^{2}\left(\boldsymbol{a}_{i}^{\perp} \cdot \boldsymbol{e}_{j}\right)\left(\boldsymbol{a}_{i}^{\perp} \cdot \boldsymbol{e}_{k}\right) \\
=\frac{1}{3\left(\ell^{2}+1\right)}\left(\begin{array}{cccccc}
2 \ell^{2} & -\sqrt{3} \ell & \ell^{2} & 0 & -\ell^{2} & \sqrt{3} \ell \\
-\sqrt{3} \ell & 2 & -\sqrt{3} \ell & 1 & 0 & -1 \\
\ell^{2} & -\sqrt{3} \ell & 2 \ell^{2} & -\sqrt{3} \ell & \ell^{2} & 0 \\
0 & 1 & -\sqrt{3} \ell & 2 & -\sqrt{3} \ell & 1 \\
-\ell^{2} & 0 & \ell^{2} & -\sqrt{3} \ell & 2 \ell^{2} & -\sqrt{3} \ell \\
\sqrt{3} \ell & -1 & 0 & 1 & -\sqrt{3} \ell & 2
\end{array}\right),
\end{gathered}
$$

so that $Q^{2}=Q$ and $Q \boldsymbol{e}_{k}=\boldsymbol{e}_{k}^{\perp}$. In addition, we have

$$
\left|\boldsymbol{e}_{k}^{\perp}\right|=\ell \sqrt{\frac{2}{3\left(\ell^{2}+1\right)}} \text { for } k=1,3,5
$$

and

$$
\left|\boldsymbol{e}_{k}^{\perp}\right|=\sqrt{\frac{2}{3\left(\ell^{2}+1\right)}} \text { for } k=2,4,6 .
$$

These projected basis vectors are shown in Fig. S10b.
The other perpendicular space is spanned by

$$
\boldsymbol{b}_{1}^{\perp}=\frac{1}{\sqrt{3}}(0,1,0,-1,0,1)
$$

and

$$
\boldsymbol{b}_{2}^{\perp}=\frac{1}{\sqrt{3}}(1,0,-1,0,1,0)
$$

produce another invariant subspace corresponding to the perpendicular space. Then the projection operator onto the perpendicular space is

$$
\begin{gathered}
R_{j k}=\sum_{i=1}^{2}\left(\boldsymbol{b}_{i}^{\perp} \cdot \boldsymbol{e}_{j}\right)\left(\boldsymbol{b}_{i}^{\perp} \cdot \boldsymbol{e}_{k}\right) \\
=\left(\begin{array}{cccccc}
\frac{1}{3} & 0 & -\frac{1}{3} & 0 & \frac{1}{3} & 0 \\
0 & \frac{1}{3} & 0 & -\frac{1}{3} & 0 & \frac{1}{3} \\
-\frac{1}{3} & 0 & \frac{1}{3} & 0 & -\frac{1}{3} & 0 \\
0 & -\frac{1}{3} & 0 & \frac{1}{3} & 0 & -\frac{1}{3} \\
\frac{1}{3} & 0 & -\frac{1}{3} & 0 & \frac{1}{3} & 0 \\
0 & \frac{1}{3} & 0 & -\frac{1}{3} & 0 & \frac{1}{3}
\end{array}\right),
\end{gathered}
$$

where $R^{2}=R$. We limit the projected points such that they satisfy $R \boldsymbol{r}=\mathbf{0}$, meaning that the rank of the higherdimensional space is essentially four.

Therefore, the vertices of the tiling are given by $\boldsymbol{r} \|=$ $\sum_{i=1}^{4} n_{i} \boldsymbol{e}_{i}^{\|}$, where $\left\{n_{i}\right\}$ is a set of four integers, and the corresponding position in the perpendicular space is given by $\boldsymbol{r}^{\perp}=\sum_{i=1}^{4} n_{i} \boldsymbol{e}_{i}^{\perp}$, where $\boldsymbol{e}_{i}^{\perp}$ is defined in Fig. S10.

However, it is convenient to express the same vertices using tiling vectors $\tilde{\boldsymbol{e}}_{i}^{\|}(i=1,2,3,4)$ shown in Fig. S11a: $\boldsymbol{r}^{\|}=\sum_{i=1}^{4} \tilde{n}_{i} \tilde{\boldsymbol{e}}_{i}^{\|}$, where $\left\{\tilde{n}_{i}\right\}$ is a set of four integers. The relation between the two sets of integers is described by

$$
\left(\begin{array}{c}
n_{1} \\
n_{2} \\
n_{3} \\
n_{4}
\end{array}\right)=\left(\begin{array}{cccc}
0 & 1 & 0 & -1 \\
2 & 0 & 1 & 0 \\
0 & 1 & 0 & 2 \\
-1 & 0 & 1 & 0
\end{array}\right)\left(\begin{array}{c}
\tilde{n}_{1} \\
\tilde{n}_{2} \\
\tilde{n}_{3} \\
\tilde{n}_{4}
\end{array}\right)
$$

The edge lengths of the triangles and rectangles are

$$
\left|\tilde{\boldsymbol{e}}_{k}^{\|}\right|=\ell \sqrt{\frac{2}{\ell^{2}+1}} \approx 1.130199
$$

and

$$
\left|\tilde{\boldsymbol{e}}_{k}^{\|}\right|=\sqrt{\frac{2}{\ell^{2}+1}} \approx 0.850088,
$$

when $\ell=(\sqrt{3}+\sqrt{39}) / 6 \approx 1.32951$ of the self-similar tiling. (Recall that in this Section, $s=1$ for conciseness.)

Finally, we note that the inflation matrix from the $m$ th generation to the $(m+1)$-generation is

$$
\left(\begin{array}{c}
n_{1}(m+1) \\
n_{2}(m+1) \\
n_{3}(m+1) \\
n_{4}(m+1)
\end{array}\right)=\left(\begin{array}{cccc}
2 & 1 & 0 & -1 \\
2 & 1 & 1 & 0 \\
0 & 1 & 2 & 2 \\
-1 & 0 & 1 & 1
\end{array}\right)\left(\begin{array}{c}
n_{1}(m) \\
n_{2}(m) \\
n_{3}(m) \\
n_{4}(m)
\end{array}\right),
$$

one of its eigenvalues being the bronze mean. 


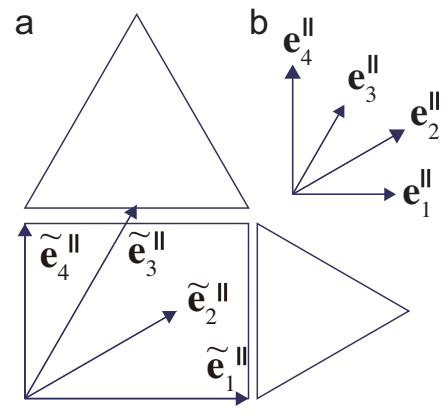

Fig. S11. a, Tiling vectors $\tilde{\boldsymbol{e}}_{i}^{\|}$whose lengths are the sides of tiles. b, Projected basis vectors $\boldsymbol{e}_{i}^{\|}$.

\section{B. Perpendicular space fluctuations}

Perpendicular space fluctuations are defined as

$$
\left(\Delta \boldsymbol{x}^{\perp}\right)^{2}=\frac{1}{N} \sum_{j=1}^{N}\left[\boldsymbol{x}^{\perp}(j)-\overline{\boldsymbol{x}^{\perp}(j)}\right]^{2},
$$

where $\overline{\boldsymbol{x}^{\perp}(j)}$ is the average of $\boldsymbol{x}^{\perp}$. From the 324391 data set, we have $\left(\Delta \boldsymbol{x}^{\perp}\right)^{2}=0.540287$ for the BMQC.

a

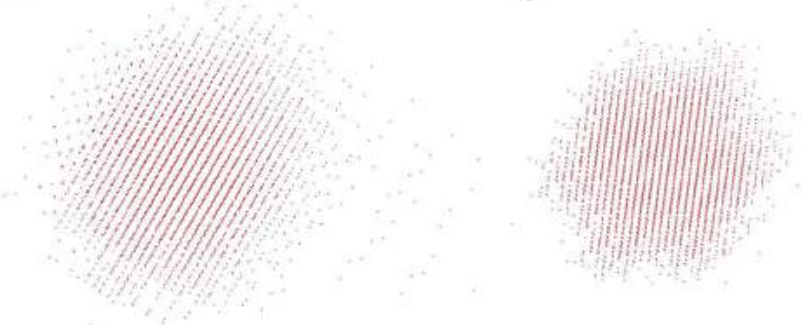

Fig. S12. a, Snapshot of the simulated HCSS BMQC in the perpendicular space corresponding to Fig. S6a for the $\lambda=2.18$ HCSS ensemble consisting of $N=4000$ particles quenched at $k_{\mathrm{B}} T / \varepsilon=0.20408$. Sizable patches of the basic approximants produce a wider distribution of points. $\mathbf{b}$, The same for Fig. S6c annealed at $k_{\mathrm{B}} T / \varepsilon=0.25$.

On the other hand, snapshots in the perpendicular space corresponding to Fig. S6a \& c are displayed in Fig. S12a \& b with perpendicular fluctuations of 0.899 and 0.617 , respectively. They are large because of sizable patches of approximants (Fig. S6b) and because of random phason fluctuations (Fig. S6d). In the quenched state (Fig. S12a), anisotropic distribution is evident, whereas the annealed state becomes compact and seems to restore 6 -fold symmetry, consistent with the random tiling hypothesis that the maximal-entropy state is the highest rotationally symmetric state, where all $N$-fold Bragg peaks should have the same intensity.

\section{Fourier transform}

Let us choose the same orientation for $\boldsymbol{r}^{\|}$and $\boldsymbol{k}^{\|}$ axes in their respective lattices, i.e., $\boldsymbol{k}_{i}=(2 \pi / a) \boldsymbol{e}_{i}$. Then the following identity holds for any pair of vectors $\boldsymbol{x}(i)=\left(\boldsymbol{x}^{\|}, \boldsymbol{x}^{\perp}, \mathbf{0}\right)$ and $\boldsymbol{k}=\left(\boldsymbol{k}^{\|}, \boldsymbol{k}^{\perp}, \mathbf{0}\right): 1=$ $\exp (i \boldsymbol{k} \cdot \boldsymbol{x}(i))=\exp \left(i \boldsymbol{k}^{\|} \cdot \boldsymbol{x}^{\|}\right) \exp \left(i \boldsymbol{k}^{\perp} \cdot \boldsymbol{x}^{\perp}\right)$. If particles' positions are described by $\delta$-functions so that the density reads $f(\boldsymbol{r} \|)=\sum_{j=1}^{N} \delta\left(\boldsymbol{r} \|-\boldsymbol{x}^{\|}(j)\right)$, then the Fourier transform of the density is given by

$$
\begin{aligned}
& \int d \boldsymbol{r}^{\|} \exp \left(-i \boldsymbol{k}^{\|} \cdot \boldsymbol{r}^{\|}\right) f\left(\boldsymbol{r}^{\|}\right)=\sum_{j=1}^{N} \exp \left(-i \boldsymbol{k}^{\|} \cdot \boldsymbol{x}^{\|}(j)\right) \\
& =\sum_{j=1}^{N} \exp \left(i \boldsymbol{k}^{\perp} \cdot \boldsymbol{x}^{\perp}(j)\right)
\end{aligned}
$$

Using 324391 points in Eq. (7), we computed peak intensities for the self-similar BMQC displayed in Fig. S13, and the value of intensities are tabulated in Table SV. The intensities are normalized by the central peak $(0,0$, $0,0)$. Peaks are indexed by four integers:

$$
\boldsymbol{k}^{\|}=\Sigma_{i} n_{i} \boldsymbol{k}_{i}^{\|}=\Sigma_{i} n_{i}(2 \pi / a) \boldsymbol{e}_{i}^{\|}
$$

As always, the smaller the $\left|\boldsymbol{k}^{\perp}\right|$, the stronger the intensities. Fig. S13 was used to create the overlaid diffraction pattern in Fig. 2e of the main text.

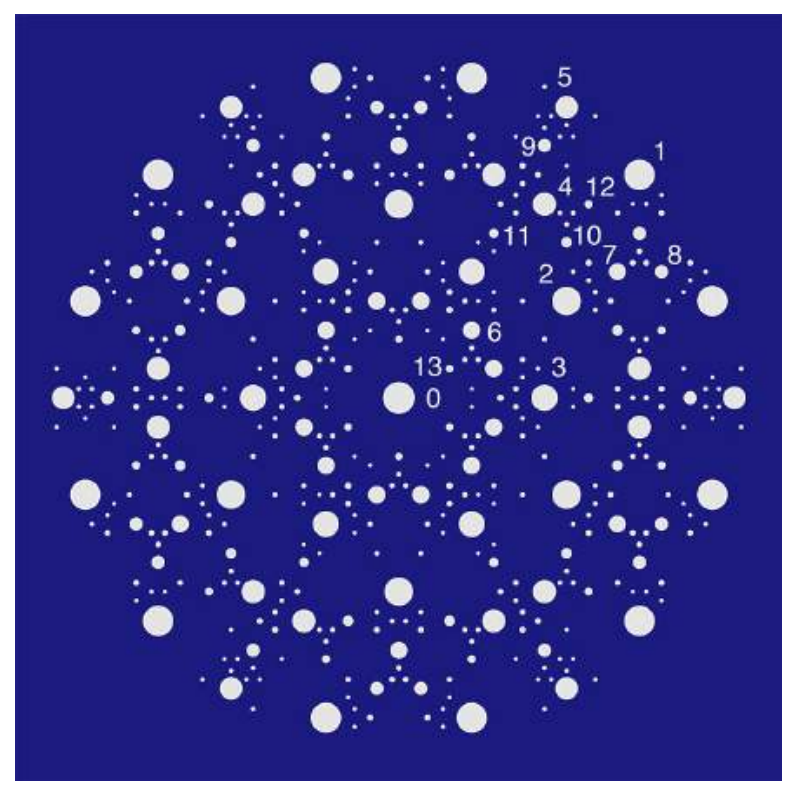

Fig. S13. Fourier transform of the BMQC computed using the higher-dimensional method with intensity $I\left(\boldsymbol{k}^{\|}\right)>0.0045$. The spot area corresponds to the value of the intensity. Peaks are indexed in Table S V. 


\begin{tabular}{|c|cccc|c|c|c|}
\hline No. & $n_{1}$ & $n_{2}$ & $n_{3}$ & $n_{3}$ & Intensity & $\left|\boldsymbol{k}^{\perp}\right|$ & $\left|\boldsymbol{k}^{\|}\right|$ \\
\hline 0 & 0 & 0 & 0 & 0 & 1.000 & 0.0 & 0.0 \\
\hline 1 & 1 & 3 & 2 & 1 & 0.896 & 0.637 & 22.934 \\
\hline 2 & 1 & 2 & 1 & 0 & 0.788 & 0.934 & 13.541 \\
\hline 3 & 0 & 1 & 1 & 1 & 0.652 & 1.241 & 10.185 \\
\hline 4 & 0 & 2 & 2 & 1 & 0.505 & 1.553 & 16.944 \\
\hline 5 & 0 & 2 & 3 & 2 & 0.475 & 1.617 & 23.454 \\
\hline 6 & 0 & 1 & 1 & 0 & 0.267 & 2.102 & 6.944 \\
\hline 7 & 1 & 3 & 1 & 0 & 0.250 & 2.150 & 17.641 \\
\hline 8 & 2 & 3 & 1 & 0 & 0.145 & 2.483 & 20.370 \\
\hline 9 & 0 & 2 & 2 & 2 & 0.143 & 2.483 & 20.370 \\
\hline 10 & 1 & 2 & 1 & 1 & 0.084 & 2.739 & 15.990 \\
\hline 11 & 0 & 1 & 2 & 1 & 0.061 & 2.859 & 13.269 \\
\hline 12 & 1 & 2 & 2 & 1 & 0.042 & 3.007 & 18.958 \\
\hline 13 & 0 & 1 & 0 & 0 & 0.034 & 3.084 & 4.100 \\
\hline
\end{tabular}

Table S V. List of peaks for the BMQC having intensity $I\left(\boldsymbol{k}^{\|}\right)>0.03$.

\section{Comparison with simulations}

In Fig. S14 we plot angular-integrated intensities

$$
I\left(k^{\|}\right)=\int I\left(\boldsymbol{k}^{\|}\right) k \mathrm{~d} \theta
$$

for the self-similar BMQC and Monte Carlo averaged intensities for simulations at $k_{\mathrm{B}} T / \varepsilon=0.25$. The positions of the strong peaks coincide with theoretical calculations.

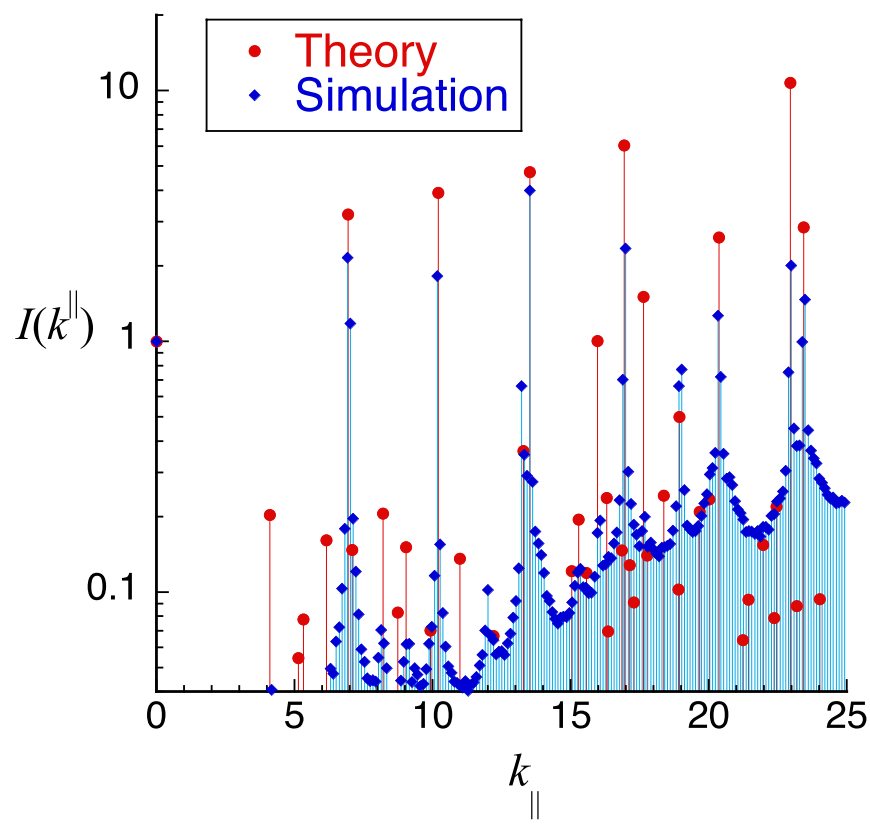

Fig. S14. Angular-integrated intensities $I\left(k^{\|}\right)$: Theory and simulation.
However, the most prominent peaks in simulations correspond to peak No. 2 in Fig. S13 and in Table S V. Diffuse wings are seen in simulations.

\section{STABILITY OF IDEAL BMQC IN VIEW OF PHASON FLUCTUATIONS}

Below we examine the stability of the ideal BMQC at finite temperatures by employing large rectangle approximants of the BMQC chosen because here periodic boundary conditions are convenient to implement. The corresponding points in the perpendicular space from the third- to fifth-generation rectangle approximants are shown in Fig. S15. Notice that the positions are corrected by $\tilde{\boldsymbol{x}}^{\perp}=\boldsymbol{x}^{\perp}-\boldsymbol{E} \boldsymbol{x}^{\|}$, where $\boldsymbol{E}$ is the linear phason strain matrix: e.g. $\boldsymbol{E}_{x x}=\partial x^{\perp} / \partial x^{\|}$for rectangles. The periodic arrays of points in the snowflake window shown in Fig. S15 imply the commensurability of the structure.
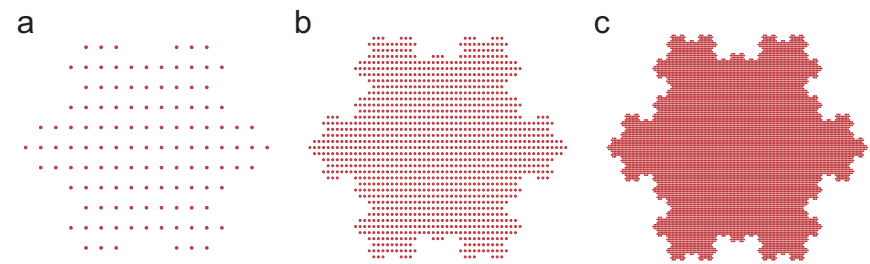

Fig. S15. Perpendicular space view of rectangle approximants. The numbers of points are $\mathbf{a}, 121, \mathbf{b}, 1319$, and $\mathbf{c}$, 14389 for the third, fourth, and fifth generation, respectively. The positions in the perpendicular space are corrected by the linear phason strain.

The question addressed here is whether HCSS particles with $\lambda=2.18$ stabilise BMQCs as random tilings or not. For this purpose, we conducted thermalisation experiments of particles in rectangle approximants with $\eta=0.600$, where a density a little higher than 0.595 is chosen so as to reduce the number of shield-like defects. Snapshots of particle positions in the perpendicular space After themalisation at $k_{\mathrm{B}} T / \varepsilon=0.20$ and $k_{\mathrm{B}} T / \varepsilon=0.25$ are shown in Fig. 4f \& $\mathrm{g}$ of the main text.

In our simulations, the system remained in the BMQC state for $5 \times 10^{8} \mathrm{MC}$ steps. Specifically, it was locked in an almost perfect-tiling state at $k_{\mathrm{B}} T / \varepsilon=0.20$ (Fig. $4 \mathrm{f}$ of the main text), and unlocked to an random-tiling state at $k_{\mathrm{B}} T / \varepsilon=0.25$ (Fig. $4 \mathrm{~g}$ of the main text). Phason fluctuations are evaluated using Eq. (6) and averaged in the MC simulations. Compared to the smaller approximants with 1319 and 121 particles, the phason fluctuations increase with system size as shown in Fig. S16, consistent with the entropic ordering mechanism ${ }^{4}$. The scaling behavior (precisely speaking the logarithmic divergence) cannot be obtained from these data, because the smaller system of 121 particles is too small. 


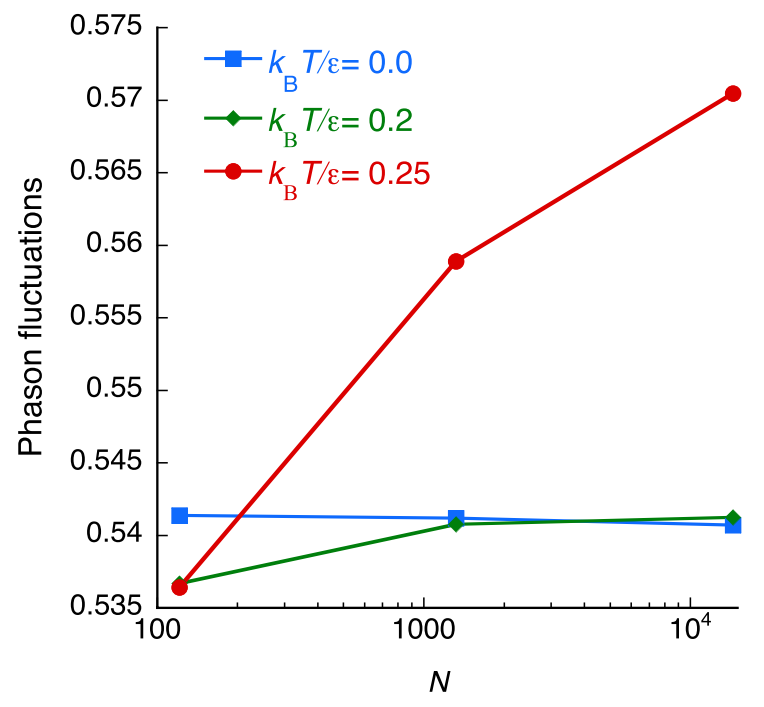

Fig. S16. Phason fluctuations as a function of system size. The numbers of particles are 121, 1319, and 14389 for the third-, fourth-, and fifth-generation rectangle approximation, respectively.

${ }^{1}$ Gummelt, P. Penrose tilings as coverings of congruent decagons. Geom. Dedic. 62, 1-17 (1996).

2 Elser, V. Indexing problems in quasicrystal diffraction. Phys. Rev. B 32, 4892-4898 (1985).

3 Jarić, M. V. Diffraction from quasicrystals: geometric structure factor. Phys. Rev. B 34, 4685-4698 (1986).

4 There exists a type of phason disorder known as the random phason. Let $\boldsymbol{v}(\boldsymbol{r})$ be a coarse-grained field of $\boldsymbol{x}^{\perp}$ around $\boldsymbol{x}$. In perfect tilings, $\boldsymbol{v}(\boldsymbol{r})=$ const. In random tilings, it is assumed that the elastic free energy $F$ from tiling configurational entropy is described by

$$
F=\frac{K}{2} \int \mathrm{d} \boldsymbol{r} \sum_{i}\left[\nabla v_{i}(\boldsymbol{r})\right]^{2} .
$$

Here we assume that the elastic constant is isotropic, that $F$ includes no energetic term like in rubber elasticity, and that the formation of a quasicrystal lowers the free energy. Dimensionality of the system is one of the important aspects. In two-dimensional quasicrystals, the thermal aver- age of random phason fluctuations is given by

$$
\left\langle\Delta \boldsymbol{v}^{2}\right\rangle \sim \frac{2 \pi}{\beta K} \ln L+\text { const. }
$$

where $\Delta v=v-\langle v\rangle$ and $L$ is system size. Consequently, the phason fluctuations diverge logarithmically ${ }^{5}$, the divergence stemming from long-wavelength fluctuations. Therefore, phason variables cannot be restricted to a finite window. In other words, two-dimensional tiling systems cannot have $\delta$-function Bragg peaks and perfect quasiperiodicity in a strict sense. In fact, for the Penrose tiling with a ground state enforced by matching rules, a Monte Carlo simulation showed that at any finite temperature, the tiling is always in the random tiling state in which phason fluctuations dominate $^{6}$. It means that even a locally perfect Penrose tiling is globally random when scaled up.

${ }^{5}$ Henley, C. L. Random Tiling Models. In Steinhardt, P. J. \& Divincenzo, D. P. (Eds.) Quasicrystals: The State of the Art, 2nd Edition, Ch. 8, (World Scientific: Singapore, 1999).

6 Tang, L.-H. \& Jarić, M. V. Equilibrium quasicrystal phase of a Penrose tiling model Phys. Rev. B 41 4524-4546 (1990). 